\title{
Toxicological evaluations of betulinic acid and ursolic acid; common constituents of Houttuynia cordata used as an anthelmintic by the Naga tribes in North-east India
}

Vijaya Mishra $^{1 *}$ D, Amar Deep Soren ${ }^{1,2}$ and Arun Kumar Yadav ${ }^{1}$

\begin{abstract}
Background: Betulinic acid (BA) and ursolic acid (UA) are two major phytoconstituents of Houttuynia cordata Thunb., (Saururaceae) which is used as an anthelmintic in the traditional medicine system of the Nagas in Nagaland, India. This study evaluates their toxic potentials using rodent models (Swiss albino mice and Wistar rats) according to the OECD (Organization for Economic Cooperation and Development) guidelines. Acute and 28-day sub-acute oral toxicity studies were conducted, and evaluations were made based on biochemical, hematological, and histopathological observations.

Results: Acute oral toxicity study revealed the oral LD50 of both the test compounds to be $>2000 \mathrm{mg} / \mathrm{kg}$ in mice. Sub-acute administration of BA at $10 \mathrm{mg} / \mathrm{kg}$ body weight (b.w.) revealed a significant increase in serum glutamic oxaloacetic transaminase (SGOT), alkaline phosphatase (ALP), urea concentrations and eosinophil and lymphocyte counts in rats. Animals administered with $10 \mathrm{mg} / \mathrm{kg}$ b.w. UA revealed elevated neutrophil count, SGOT, ALP, and urea concentrations, whereas white blood cells (WBC), lymphocyte, and platelet counts were found to be low. Histopathological examinations of body organs revealed alterations in the architecture of the liver, kidney, and spleen tissues. Notably, all these alterations were recoverable as evident in the satellite group, indicating a recovering pattern from the toxic effects caused by the oral administration of these phytocompounds.
\end{abstract}

Conclusion: Although UA and BA possess several therapeutic properties, their long-term usage can cause mild toxicity in their users. This study also paves way for evaluating the optimum effective and safe dose of these phytocompounds.

Keywords: Betulinic acid, Phytocompounds, Toxicity, Ursolic acid

\footnotetext{
*Correspondence: mishra.vijaya@yahoo.co.in

'Department of Zoology, North-Eastern Hill University, Shillong, Meghalaya

793022, India

Full list of author information is available at the end of the article
}

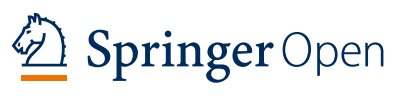

(c) The Author(s). 2021 Open Access This article is licensed under a Creative Commons Attribution 4.0 International License, which permits use, sharing, adaptation, distribution and reproduction in any medium or format, as long as you give appropriate credit to the original author(s) and the source, provide a link to the Creative Commons licence, and indicate if changes were made. The images or other third party material in this article are included in the article's Creative Commons licence, unless indicated otherwise in a credit line to the material. If material is not included in the article's Creative Commons licence and your intended use is not permitted by statutory regulation or exceeds the permitted use, you will need to obtain permission directly from the copyright holder. To view a copy of this licence, visit http://creativecommons.org/licenses/by/4.0/. 


\section{Background}

Natural products serve as raw materials of drugs, and the isolated bioactive compounds have been the foundation for several known pharmaceuticals. Almost 50\% of all medicines today are either derived or altered form of the active phytocompounds of therapeutic plants [1], and studies on such novel phytocompounds to treat various ailments are a rapidly growing area of research [2]. Several compounds isolated from plants include artemisinin used in malarial treatment, nitisinone used in the treatment of tyrosinaemia, galantamine for Alzheimer's, apomorphine used in Parkinson's disease, tiotropium used in chronic obstructive pulmonary disease, and dronabinol, cannabidiol, and capsaicin used as pain relievers [3], and compounds such as paclitaxel, vincristine, vinblastine, and camptothecin are known to possess anticancer agents $[4,5]$.

Plants produce toxic secondary metabolites as natural defense from adverse conditions, and toxicity testing can reveal the risks that may be associated thereby, avoiding potential harmful effects when used as medicine [6]. Unregulated consumption of the medicinal plants or their compounds has been an essential part of various studies that reveal the effects, doses, and toxicity exhibiting mild to lethal effects [7]. Several compounds such as capsaicin, cycasin, genistein, and ptaquiloside used as food/ supplements have been found to be carcinogenic [8]. Many isolated and derived phytocompounds have been evaluated for their potential side effects via acute and chronic studies [9-11]. Several compounds possessing anthelmintic efficacy such as doramectin, eprinomectin, and selamectin have also been tested for their potential toxic effects [12].

Betulinic acid (BA) is a naturally occurring pentacyclic lupane-type triterpene [13] which has been used in folk medicine for centuries [14]. It is known to possess antiviral [15], anti-malarial [16], anti-inflammatory [17], and anthelmintic activity against Caenorhabditis elegans [18]. A toxicity study on betulin, a precursor for BA, revealed that its sub-acute oral administration did not cause toxicity in rats and dogs [19]. Also, it has been established that BA delivered in liposomes does not cause systemic toxicity in mice [20].

Ursolic acid (UA) is a pentacyclic triterpenoid (saponin) present in food and plants which is known to protect hepatocytes from ethanol toxicity [21]. It has been reported to possess anti-bacterial, hepatoprotective, immunomodulatory, anti-proliferative [22], and antiinflammatory activities [23]. It has been known to possess anti-cancer properties as well [24]. UA is also reported to possess anti-filarial effect [25] and is effective in the treatment of Alzheimer's disease [26]. A toxicity study revealed that administration of UA at a dose below $1000 \mathrm{mg} / \mathrm{kg} /$ day does not cause any toxic effects on its users [27]. However, a clinical study revealed that administration of UA liposomes causes hepatotoxicity and diarrhea [28].

BA and UA are also reported to possess nematicidal efficacy against Trichostrongylus colubriformis, Haemonchus contortus, and C. elegans [29]. They have also been proven to possess anthelmintic efficacy against Syphacia obvelata [30] and Hymenolepis diminuta [31]. BA and UA are major constituents in Houttuynia cordata Thunb., (Saururaceae) which is used as an anthelmintic in the traditional medicine system of the Nagas in Nagaland, India [32]. Since their anthelmintic potentials have been already reported by several authors, this study evaluates their toxic potentials using suitable animal models.

\section{Material and methods}

\section{Experimental compounds}

BA and UA were obtained from Sigma-Aldrich, Mumbai, and acetaminophen (APAP) was obtained from SD Fine Chemicals Limited, Mumbai.

\section{Experimental animals}

Five healthy female Swiss mice, about $7-8$ weeks of age and weighing about 25 to $30 \mathrm{~g}$, were used for acute toxicity studies, and 40 female and 40 male rats of Wistar strain, about 7-9 weeks of age and weighing about 150 to $200 \mathrm{~g}$, were used for sub-acute toxicity studies. All the animals were kept in separate cages and were acclimatized to laboratory conditions. They had ad libitum access to food and water. All experiments on laboratory animals were approved by the Institutional Ethics Committee (Animal models). Also, all experiments on animals comply with the ARRIVE (Animal Research: Reporting of In Vivo Experiments) guidelines.

\section{Limit test for acute oral toxicity}

Limit test was performed on 5 female Swiss mice as per the Organization for Economic Cooperation and Development (OECD) guideline 423 [33]. In brief, a single oral dose of $2000 \mathrm{mg} / \mathrm{kg}$ body weight (b.w.) of test compounds in $0.5 \mathrm{ml}$ vehicle phosphate-buffered saline $(\mathrm{PBS})+$ few drops of $1 \%$ dimethyl sulphoxide (DMSO) was given to a female Swiss mouse and observed for mortality. If the animal survived, four additional animals were administered the same dose at an interval of $48 \mathrm{~h}$. Animals were observed for adverse signs of toxicity or mortality during the first $30 \mathrm{~min}$ and then periodically during the first $24 \mathrm{~h}$ (every $12 \mathrm{~h}$ with special attention given during the first $4 \mathrm{~h}$ ). The animals were kept under observation for 2 weeks to observe for any signs of distress, toxicity, or mortality. 
The LD50 was predicted to be greater than $2000 \mathrm{mg} /$ $\mathrm{kg}$, if three or more animals survived.

\section{Sub-acute toxicity study}

The 28-day oral toxicity study was performed as per the OECD guideline 407 [34]. Accordingly, three different doses were selected for the study. Dose selection was based on the efficacy of different phytocompounds in the in vivo study. Doses that showed optimum efficacy and could show any signs of toxicity at the highest test dose were selected for the study. Hence, animals were dosed 1, 5, and $10 \mathrm{mg} / \mathrm{kg}$ b.w. of BA and UA. Animals were divided into eight groups $(n=10,5 / \mathrm{sex})$. Group 1 served as the negative control and was orally fed with the $0.5 \mathrm{ml}$ vehicle (PBS+DMSO). Groups 2, 3, and 4 were orally administered 1,5 , and $10 \mathrm{mg} / \mathrm{kg}$ b.w. doses of BA and UA dissolved in DMSO. Group 5 served as the positive control and was administered APAP at 50 $\mathrm{mg} / \mathrm{kg}$ b.w. Groups 6,7 , and 8 served as the satellite control, satellite group which received the highest concentration of phytocompound (10 mg/kg b.w.), and APAP group (50 mg/kg b.w.), respectively. Groups 6, 7, and 8 were kept for an additional 2 weeks to observe for any reversion or persistence from the toxic effects, if any. Animals were checked daily for general clinical signs of toxic effects or mortality, and weekly, their body weight and food and water consumption were measured. Animals from groups 1-5 were sacrificed by administering anesthesia on the 29th day and groups 6-8 were sacrificed on the 43rd day. Before the administration of anesthesia, animals were refrained from food but not water, and atropine $(0.02$ $\mathrm{mg} / \mathrm{kg}$; s.c) was given as prescribed anesthetic barbiturate i.p. Blood samples $(2 \mathrm{ml})$ were collected from the animals through cardiac puncture and processed for hematological and serum biochemical analysis. The animals were then sacrificed by cervical dislocation quickly to ascertain less anxiety and painless death. The liver, heart, spleen, kidney, brain, adrenal glands, testes, and ovaries were removed to analyze the relative organ weights (ROW). Vital organs were washed with distilled water and preserved in Bouin's fixative to carry out histopathological studies. Hematological analysis was performed using Alfa Basic, Swelab, Germany, a semi-auto cell counter, and biochemical analysis was performed using Synergy BIO 1904C, Euro Diagnostic Systems Pvt. Ltd., India, a semi-auto biochemistry analyzer.

\section{Statistical analysis}

All the experimental data are represented as mean \pm standard error of mean (SEM). Data were analyzed by one-way analysis of variance (ANOVA), comprising of Bonferroni's test. The $p$ value $\leq 0.05$ was considered to be statistically significant. All the statistical calculations were done using Origin Pro 8.

Table 1 Effect of sub-acute oral administration of BA and APAP on body weight (g) of Wistar rats ( $n=5 / \mathrm{sex}$ )

\begin{tabular}{|c|c|c|c|c|c|c|c|c|}
\hline \multirow[t]{2}{*}{ Week } & \multirow{2}{*}{$\begin{array}{l}\text { G-I } \\
\text { Control }\end{array}$} & \multirow{2}{*}{$\begin{array}{l}\text { G-II } \\
\text { BA } \\
1 \mathrm{mg} / \mathrm{kg}\end{array}$} & \multirow{2}{*}{$\begin{array}{l}\text { G-III } \\
\text { BA } \\
5 \mathrm{mg} / \mathrm{kg}\end{array}$} & \multirow{2}{*}{$\begin{array}{l}\text { G-IV } \\
\text { BA } \\
10 \mathrm{mg} / \mathrm{kg}\end{array}$} & \multirow{2}{*}{$\begin{array}{l}\text { G-V } \\
\text { APAP } \\
50 \mathrm{mg} / \mathrm{kg}\end{array}$} & \multicolumn{3}{|c|}{ Satellite groups } \\
\hline & & & & & & $\begin{array}{l}\mathrm{G}-\mathrm{VI} \\
\text { Control }\end{array}$ & $\begin{array}{l}\text { G-VII } \\
\text { BA } \\
10 \mathrm{mg} / \mathrm{kg}\end{array}$ & $\begin{array}{l}\text { G-VIII } \\
\text { APAP } \\
50 \mathrm{mg} / \mathrm{kg}\end{array}$ \\
\hline \multicolumn{9}{|l|}{ Female } \\
\hline Wo & $196.4 \pm 3.22$ & $194.2 \pm 2.65$ & $193.2 \pm 2.24$ & $194.6 \pm 2.54$ & $193.6 \pm 1.13$ & $195.6 \pm 2.83$ & $198.6 \pm 1.43$ & $200.8 \pm 1.24$ \\
\hline W1 & $196.2 \pm 4.21$ & $196.7 \pm 2.48$ & $192.2 \pm 2.80$ & $197.8 \pm 0.80$ & $188.5 \pm 0.44$ & $200.6 \pm 1.20$ & $195.3 \pm 0.63$ & $202.2 \pm 1.18$ \\
\hline W2 & $200.2 \pm 3.39$ & $194.8 \pm 2.47$ & $193.6 \pm 1.66$ & $196.2 \pm 0.58$ & $186.2 \pm 0.37$ & $203.4 \pm 1.28$ & $195.2 \pm 0.58$ & $200.4 \pm 1.20$ \\
\hline W3 & $203.2 \pm 3.11$ & $193.8 \pm 1.15^{\mathrm{a}}$ & $191.2 \pm 1.70^{\mathrm{a}}$ & $195.8 \pm 0.66^{\mathrm{a}}$ & $182.2 \pm 0.37$ & $205.4 \pm 1.28$ & 194.4. \pm 0.50 & $198.4 \pm-0.87^{c}$ \\
\hline W4 & $204.8 \pm 3.07$ & $192.8 \pm 2.47^{\mathrm{a}}$ & $190.6 \pm 1.86^{a}$ & $193.8 \pm 0.66^{\mathrm{a}}$ & $178.6 \pm 0.31^{\mathrm{a}}$ & $207.2 \pm 1.34$ & $192.2 \pm 0.58^{c}$ & $196.8 \pm 0.58$ \\
\hline W5 & & & & & & $209.1 \pm 0.37$ & $195.5 \pm 0.70$ & $199.4 \pm 0.50$ \\
\hline W6 & & & & & & $210.2 \pm 1.31$ & $197.4 \pm 0.81$ & $202.6 \pm 0.42^{c}$ \\
\hline \multicolumn{9}{|l|}{ Male } \\
\hline Wo & $201.8 \pm 0.86$ & $204.8 \pm 2.61$ & $207.2 \pm 0.89$ & $205.2 \pm 0.44$ & $203.2 \pm 0.48$ & $197.6 \pm 2.81$ & $200.4 \pm 0.30$ & $203.2 \pm 0.70$ \\
\hline W1 & $204.2 \pm 0.63$ & $205.4 \pm 2.73$ & $207.6 \pm 0.81$ & $204.2 \pm 0.37$ & $200.6 \pm 0.24$ & $200.4 \pm 3.92$ & $198.4 \pm 1.50$ & $203.8 \pm 1.73$ \\
\hline W2 & $205.6 \pm 0.40$ & $204.2 \pm 0.91$ & $205.8 \pm 0.58$ & $202.8 \pm 0.45$ & $195.6 \pm 0.24$ & $203.6 \pm 3.74$ & $196.6 \pm 1.50^{c}$ & $201.6 \pm 2.81$ \\
\hline W3 & $207.6 \pm 1.50$ & $204.8 \pm 0.96$ & $204.5 \pm 0.54$ & $200.2 \pm 0.37^{\mathrm{a}}$ & $189.6 \pm 0.24^{a}$ & $205.8 \pm 1.58$ & $197.4 \pm 1.25^{\mathrm{C}}$ & $199.2 \pm 0.86$ \\
\hline W4 & $209.2 \pm 2.66$ & $202.8 \pm 0.96^{\mathrm{a}}$ & $202.8 \pm 0.66^{a}$ & $198.6 \pm 0.50^{b}$ & $185.4 \pm 0.24^{a}$ & $208.2 \pm 1.54$ & $196.2 \pm 0.37^{c}$ & $197.2 \pm 2.73$ \\
\hline W5 & & & & & & $210.6 \pm 2.81$ & $198.8 \pm 0.48$ & $199.6 \pm 0.74$ \\
\hline W6 & & & & & & $212.2 \pm 2.66$ & $200.4 \pm 2.81$ & $202.2 \pm 2.54$ \\
\hline
\end{tabular}

Data are expressed as mean \pm SEM; treatment: G-II to G-IV $=4$ weeks, G-V to G-VI $=6$ weeks; W0: week before treatment; W1-W6: weeks post-treatment ${ }^{\mathrm{a}} p<0.05,{ }^{\mathrm{b}} p<0.01$, vs control; ${ }^{c} p<0.05$, vs satellite control, one-way ANOVA 
Table 2 Effects of sub-acute oral administration of UA and APAP on body weight $(\mathrm{g})$ of Wistar rats $(n=5 / \mathrm{sex})$

\begin{tabular}{|c|c|c|c|c|c|c|c|c|}
\hline \multirow[t]{2}{*}{ Week } & \multirow{2}{*}{$\begin{array}{l}\text { G-I } \\
\text { Control }\end{array}$} & \multirow{2}{*}{$\begin{array}{l}\text { G-II } \\
\text { UA } \\
1 \text { mg/kg }\end{array}$} & \multirow{2}{*}{$\begin{array}{l}\text { G-III } \\
\text { UA } \\
5 \mathrm{mg} / \mathrm{kg}\end{array}$} & \multirow{2}{*}{$\begin{array}{l}\text { G-IV } \\
\text { UA } \\
10 \mathrm{mg} / \mathrm{kg}\end{array}$} & \multirow{2}{*}{$\begin{array}{l}\text { G-V } \\
\text { APAP } \\
50 \mathrm{mg} / \mathrm{kg}\end{array}$} & \multicolumn{3}{|c|}{ Satellite groups } \\
\hline & & & & & & $\begin{array}{l}\text { G-VI } \\
\text { Control }\end{array}$ & $\begin{array}{l}\text { G-VII } \\
\text { UA } \\
10 \mathrm{mg} / \mathrm{kg}\end{array}$ & $\begin{array}{l}\text { G-VIII } \\
\text { APAP } \\
50 \mathrm{mg} / \mathrm{kg}\end{array}$ \\
\hline \multicolumn{9}{|l|}{ Female } \\
\hline wo & $196.2 \pm 2.88$ & $198.2 \pm 2.97$ & $197.4 \pm 1.77$ & $197.2 \pm 3.27$ & $199.6 \pm 3.51$ & $197.2 \pm 3.11$ & $197.2 \pm 1.72$ & $195.8 \pm 3.14$ \\
\hline W1 & $200.6 \pm 0.60$ & $200.2 \pm 1.57$ & $198.8 \pm 1.56$ & $197.6 \pm 1.72$ & $197.2 \pm 1.24$ & $202.2 \pm 0.66$ & $199.6 \pm 1.43$ & $197.7 \pm 1.70$ \\
\hline W2 & $203.4 \pm 0.50$ & $199.4 \pm 1.50$ & $198.0 \pm 1.36$ & $196.2 \pm 1.52$ & $195.8 \pm 1.11$ & $204.2 \pm 0.70$ & $197.8 \pm 1.28$ & $196.2 \pm 1.70$ \\
\hline W3 & $205.4 \pm 0.50$ & $199.8 \pm 1.31$ & $197.2 \pm 1.24^{\mathrm{a}}$ & $196.2 \pm 1.50^{\mathrm{a}}$ & $192.6 \pm 1.02^{\mathrm{a}}$ & $205.0 \pm 0.70$ & $193.4 \pm 0.92$ & $192.2 \pm 1.70$ \\
\hline W4 & $207.2 \pm 0.31$ & $199.5 \pm 1.14$ & $196.8 \pm 1.01^{\mathrm{a}}$ & $194.4 \pm 1.20^{\mathrm{a}}$ & $192.4 \pm 0.92$ & $206.6 \pm 0.50$ & $192.6 \pm 1.07$ & $190.6 \pm 1.50$ \\
\hline W5 & & & & & & $207.6 \pm 0.50$ & $193.0 \pm 0.89$ & $190.4 \pm 1.56$ \\
\hline W6 & & & & & & $209.2 \pm 0.66$ & $194.8 \pm 0.98$ & $197.2 \pm 1.78$ \\
\hline \multicolumn{9}{|l|}{ Male } \\
\hline Wo & $199.8 \pm 2.81$ & $200.2 \pm 0.82$ & $201.4 \pm 1.07$ & $201.8 \pm 1.35$ & $202.2 \pm 1.59$ & $202.6 \pm 0.70$ & $201.6 \pm 1.26$ & $202.6 \pm 1.32$ \\
\hline W1 & $205.6 \pm 1.56$ & $200.2 \pm 0.86$ & $200.8 \pm 1.06$ & $200.2 \pm 0.83$ & $201.2 \pm 1.77$ & $203.4 \pm 0.50$ & $200.3 \pm 1.22$ & $201.2 \pm 1.22$ \\
\hline W2 & $209.4 \pm 0.08$ & $199.2 \pm 0.73$ & $199.8 \pm 1.06$ & $199.5 \pm 0.83$ & $199.8 \pm 1.56$ & $204.4 \pm 0.50$ & $199.4 \pm 0.86$ & $198.6 \pm 1.07$ \\
\hline W3 & $210.8 \pm 1.49$ & $199.4 \pm 0.54$ & $198.4 \pm 1.04$ & $198.8 \pm 0.66$ & $198.4 \pm 1.43^{\mathrm{a}}$ & $205.4 \pm 0.50$ & $198.2 \pm 0.86$ & $197.6 \pm 1.07$ \\
\hline W4 & $212.8 \pm 1.68$ & $198.2 \pm 0.58$ & $197.5 \pm 1.06^{\mathrm{a}}$ & $196.2 \pm 0.54^{a}$ & $197.8 \pm 1.24^{\mathrm{a}}$ & $206.6 \pm 0.50$ & $197.4 \pm 1.04$ & $195.8 \pm 1.31$ \\
\hline W5 & & & & & & $207.6 \pm 0.50$ & $198.2 \pm 1.11$ & $198.4 \pm 1.14$ \\
\hline W6 & & & & & & $209.2 \pm 0.37$ & $199.8 \pm 1.06$ & $201.8 \pm 1.25$ \\
\hline
\end{tabular}

Data are expressed as mean \pm SEM; treatment: G-II to G-IV = 4 weeks, G-V to G-VI = 6 weeks; W0: week before treatment; W1-W6: weeks post-treatment ${ }^{\mathrm{a}} p<0.05$, vs control; ${ }^{\mathrm{b}} p<0.05$ vs satellite control, one-way ANOVA

Table 3 Effects of sub-acute oral administration of BA and APAP on ROW of Wistar rats ( $n=5 / \mathrm{sex}$ )

\begin{tabular}{|c|c|c|c|c|c|c|c|c|}
\hline \multirow[t]{2}{*}{ Organ } & \multirow{2}{*}{$\begin{array}{l}\text { G-I } \\
\text { Control }\end{array}$} & \multirow{2}{*}{$\begin{array}{l}\text { G-II } \\
\text { BA } \\
1 \mathrm{mg} / \mathrm{kg}\end{array}$} & \multirow{2}{*}{$\begin{array}{l}\text { G-III } \\
\text { BA } \\
5 \mathrm{mg} / \mathrm{kg}\end{array}$} & \multirow{2}{*}{$\begin{array}{l}\text { G-IV } \\
\text { BA } \\
10 \mathrm{mg} / \mathrm{kg}\end{array}$} & \multirow{2}{*}{$\begin{array}{l}\text { G-V } \\
\text { APAP } \\
50 \mathrm{mg} / \mathrm{kg}\end{array}$} & \multicolumn{3}{|c|}{ Satellite groups } \\
\hline & & & & & & $\begin{array}{l}\text { G-VI } \\
\text { Control }\end{array}$ & $\begin{array}{l}\text { G-VII } \\
\text { BA } \\
10 \mathrm{mg} / \mathrm{kg}\end{array}$ & $\begin{array}{l}\text { G-VIII } \\
\text { APAP } \\
50 \mathrm{mg} / \mathrm{kg}\end{array}$ \\
\hline \multicolumn{9}{|l|}{ Female } \\
\hline Heart & $0.49 \pm 0.05$ & $0.49 \pm 0.04$ & $0.64 \pm 0.06$ & $0.62 \pm 0.06$ & $0.61 \pm 0.03^{c}$ & $0.63 \pm 0.07$ & $0.65 \pm 0.02$ & $0.53 \pm 0.04$ \\
\hline Liver & $5.69 \pm 0.38$ & $5.96 \pm 0.56$ & $5.44 \pm 0.49$ & $5.02 \pm 0.30^{\mathrm{a}}$ & $6.82 \pm 0.83^{c}$ & $5.92 \pm 0.06$ & $6.38 \pm 0.25$ & $5.11 \pm 0.60$ \\
\hline Brain & $1.03 \pm 0.01$ & $0.02 \pm 0.00$ & $1.05 \pm 0.02$ & $1.02 \pm 0.01$ & $1.00 \pm 0.00$ & $1.02 \pm 0.01$ & $1.04 \pm 0.02$ & $1.02 \pm 0.05$ \\
\hline Spleen & $0.43 \pm 0.05$ & $0.40 \pm 0.03$ & $0.47 \pm 0.05$ & $0.35 \pm 0.02^{b}$ & $0.67 \pm 0.04^{c}$ & $0.48 \pm 0.04$ & $0.51 \pm 0.05$ & $0.52 \pm 0.02$ \\
\hline Adrenal & $0.02 \pm 0.00$ & $0.03 \pm 0.00$ & $0.02 \pm 0.00$ & $0.02 \pm 0.00$ & $0.03 \pm 0.00$ & $0.03 \pm 0.00$ & $0.02 \pm 0.00$ & $0.03 \pm 0.00$ \\
\hline Kidney (L) & $0.53 \pm 0.03$ & $0.54 \pm 0.06$ & $0.65 \pm 0.02$ & $0.59 \pm 0.04$ & $0.45 \pm 0.02$ & $0.57 \pm 0.63$ & $0.58 \pm 0.04$ & $0.49 \pm 0.02$ \\
\hline Kidney (R) & $0.58 \pm 0.01$ & $0.53 \pm 0.06$ & $0.55 \pm 0.04$ & $0.60 \pm 0.09$ & $0.49 \pm 0.02$ & $0.74 \pm 0.02$ & $0.69 \pm 0.02$ & $0.51 \pm 0.00$ \\
\hline Ovary & $0.06 \pm 0.00$ & $0.07 \pm 0.00$ & $0.07 \pm 0.00$ & $0.06 \pm 0.00$ & $0.06 \pm 0.00$ & $0.06 \pm 0.00$ & $0.06 \pm 0.00$ & $0.06 \pm 0.00$ \\
\hline \multicolumn{9}{|l|}{ Male } \\
\hline Heart & $0.54 \pm 0.06$ & $0.52 \pm 0.08$ & $0.76 \pm 0.07$ & $0.65 \pm 0.10$ & $0.69 \pm 0.05$ & $0.66 \pm 0.08$ & $0.72 \pm 0.22$ & $0.60 \pm 0.06$ \\
\hline Liver & $5.72 \pm 0.34$ & $6.13 \pm 0.42$ & $6.38 \pm 0.58$ & $5.46 \pm 0.22^{b}$ & $6.76 \pm 1.02$ & $6.25 \pm 0.43$ & $6.86 \pm 0.19$ & $6.12 \pm 0.56$ \\
\hline Brain & $1.03 \pm 0.01$ & $1.02 \pm 0.01$ & $1.05 \pm 0.01$ & $1.03 \pm 0.01$ & $1.01 \pm 0.00$ & $1.03 \pm 0.03$ & $1.10 \pm 0.07$ & $1.05 \pm 0.05$ \\
\hline Spleen & $0.45 \pm 0.03$ & $0.43 \pm 0.03$ & $0.56 \pm 0.06$ & $0.35 \pm 0.05^{b}$ & $0.67 \pm 0.04^{c}$ & $0.53 \pm 0.05$ & $0.55 \pm 0.03$ & $0.61 \pm 0.01$ \\
\hline Adrenal & $0.02 \pm 0.00$ & $0.02 \pm 0.00$ & $0.02 \pm 0.00$ & $0.03 \pm 0.00$ & $0.03 \pm 0.00$ & $0.02 \pm 0.00$ & $0.02 \pm 0.00$ & $0.02 \pm 0.00$ \\
\hline Kidney (L) & $0.49 \pm 0.02$ & $0.53 \pm 0.03$ & $0.70 \pm 0.03$ & $0.65 \pm 0.07$ & $0.54 \pm 0.02$ & $0.64 \pm 0.05$ & $0.60 \pm 0.03$ & $0.56 \pm 0.31$ \\
\hline Kidney (R) & $0.52 \pm 0.02$ & $0.50 \pm 0.07$ & $0.61 \pm 0.07$ & $0.60 \pm 0.08$ & $0.60 \pm 0.02$ & $0.76 \pm 0.03$ & $0.69 \pm 0.02$ & $0.54 \pm 0.02$ \\
\hline Testis (L) & $1.07 \pm 0.04$ & $1.07 \pm 0.02$ & $1.06 \pm 0.04$ & $1.08 \pm 0.04$ & $1.00 \pm 0.04$ & $1.07 \pm 0.03$ & $1.07 \pm 0.01$ & $1.05 \pm 0.00$ \\
\hline Testis (R) & $0.83 \pm 0.26$ & $0.78 \pm 0.18$ & $0.62 \pm 0.28$ & $0.62 \pm 0.15$ & $0.72 \pm 0.21$ & $0.81 \pm 0.28$ & $0.71 \pm 0.12$ & $0.68 \pm 0.18$ \\
\hline
\end{tabular}


Table 4 Effects of sub-acute oral administration of UA and APAP on relative organ ROW of Wistar rats $(n=5 /$ sex)

\begin{tabular}{|c|c|c|c|c|c|c|c|c|}
\hline \multirow[t]{2}{*}{ Organ } & \multirow{2}{*}{$\begin{array}{l}\text { G-I } \\
\text { Control }\end{array}$} & \multirow{2}{*}{$\begin{array}{l}\text { G-II } \\
\text { UA } \\
1 \mathrm{mg} / \mathrm{kg}\end{array}$} & \multirow{2}{*}{$\begin{array}{l}\text { G-III } \\
\text { UA } \\
5 \mathrm{mg} / \mathrm{kg}\end{array}$} & \multirow{2}{*}{$\begin{array}{l}\text { G-IV } \\
\text { UA } \\
10 \mathrm{mg} / \mathrm{kg}\end{array}$} & \multirow{2}{*}{$\begin{array}{l}\text { G-V } \\
\text { APAP } \\
50 \mathrm{mg} / \mathrm{kg}\end{array}$} & \multicolumn{3}{|c|}{ Satellite groups } \\
\hline & & & & & & $\begin{array}{l}\text { G-VI } \\
\text { Control }\end{array}$ & $\begin{array}{l}\text { G-VII } \\
\text { UA } \\
10 \mathrm{mg} / \mathrm{kg}\end{array}$ & $\begin{array}{l}\text { G-VIII } \\
\text { APAP } \\
50 \mathrm{mg} / \mathrm{kg}\end{array}$ \\
\hline \multicolumn{9}{|l|}{ Female } \\
\hline Heart & $0.57 \pm 0.01$ & $0.67 \pm 0.05$ & $0.68 \pm 0.06$ & $0.65 \pm 0.08$ & $0.62 \pm 0.06$ & $0.59 \pm 0.13$ & $0.62 \pm 0.07$ & $0.77 \pm 0.03$ \\
\hline Liver & $5.81 \pm 0.21$ & $5.66 \pm 0.69$ & $5.28 \pm 0.52$ & $4.93 \pm 0.55^{\mathrm{a}}$ & $6.82 \pm 1.05^{b}$ & $5.76 \pm 0.38$ & $5.82 \pm 0.55$ & $5.55 \pm 0.31$ \\
\hline Brain & $1.09 \pm 0.02$ & $1.02 \pm 0.02$ & $1.02 \pm 0.02$ & $1.02 \pm 0.01$ & $0.99 \pm 0.01$ & $0.99 \pm 0.00$ & $0.99 \pm 0.00$ & $0.99 \pm 0.00$ \\
\hline Spleen & $0.49 \pm 0.03$ & $0.42 \pm 0.05$ & $0.45 \pm 0.06$ & $0.35 \pm 0.05^{a}$ & $0.71 \pm 0.05^{\mathrm{a}}$ & $0.46 \pm 0.05$ & $0.39 \pm 0.08$ & $0.43 \pm 0.07$ \\
\hline Adrenal & $0.03 \pm 0.00$ & $0.03 \pm 0.00$ & $0.02 \pm 0.00$ & $0.02 \pm 0.00$ & $0.02 \pm 0.00$ & $0.03 \pm 0.00$ & $0.03 \pm 0.00$ & $0.03 \pm 0.00$ \\
\hline Kidney (L) & $0.58 \pm 0.10$ & $0.71 \pm 0.02$ & $0.63 \pm 0.04$ & $0.77 \pm 0.04$ & $0.73 \pm 0.09$ & $0.57 \pm 0.06$ & $0.55 \pm 0.09$ & $0.57 \pm 0.02$ \\
\hline Kidney (R) & $0.58 \pm 0.12$ & $0.73 \pm 0.01$ & $0.73 \pm 0.04$ & $0.72 \pm 0.08$ & $0.71 \pm 0.12$ & $0.66 \pm 0.03$ & $0.73 \pm 0.04$ & $0.57 \pm 0.01$ \\
\hline Ovary & $0.06 \pm 0.00$ & $0.06 \pm 0.00$ & $0.06 \pm 0.01$ & $0.06 \pm 0.00$ & $0.06 \pm 0.00$ & $0.06 \pm 0.00$ & $0.06 \pm 0.00$ & $0.06 \pm 0.00$ \\
\hline \multicolumn{9}{|l|}{ Male } \\
\hline Heart & $0.61 \pm 0.03$ & $0.65 \pm 0.09$ & $0.69 \pm 0.10$ & $0.72 \pm 0.09$ & $0.62 \pm 0.02$ & $0.62 \pm 0.09$ & $0.62 \pm 0.08$ & $0.77 \pm 0.04$ \\
\hline Liver & $6.46 \pm 0.15$ & $6.55 \pm 0.95$ & $6.21 \pm 0.18$ & $5.76 \pm 0.50^{\mathrm{a}}$ & $7.13 \pm 1.20^{c}$ & $6.19 \pm 1.09$ & $6.51 \pm 0.80$ & $7.23 \pm 0.57$ \\
\hline Brain & $1.28 \pm 0.10$ & $1.03 \pm 0.01$ & $1.05 \pm 0.02$ & $1.01 \pm 0.01$ & $1.01 \pm 0.01$ & $1.01 \pm 0.02$ & $1.02 \pm 0.01$ & $1.01 \pm 0.01$ \\
\hline Spleen & $0.44 \pm 0.03$ & $0.48 \pm 0.06$ & $0.34 \pm 0.03$ & $0.32 \pm 0.04^{\mathrm{a}}$ & $0.76 \pm 0.12^{\mathrm{a}}$ & $0.49 \pm 0.07$ & $0.48 \pm 0.07$ & $0.53 \pm 0.08$ \\
\hline Adrenal & $0.03 \pm 0.00$ & $0.03 \pm 0.00$ & $0.03 \pm 0.00$ & $0.02 \pm 0.00$ & $0.02 \pm 0.00$ & $0.02 \pm 0.00$ & $0.02 \pm 0.00$ & $0.03 \pm 0.00$ \\
\hline Kidney (L) & $0.60 \pm 0.14$ & $0.72 \pm 0.01$ & $0.74 \pm 0.03$ & $0.70 \pm 0.02$ & $0.77 \pm 0.02$ & $0.66 \pm 0.09$ & $0.63 \pm 0.04$ & $0.55 \pm 0.09$ \\
\hline Kidney (R) & $0.56 \pm 0.10$ & $0.78 \pm 0.01$ & $0.72 \pm 0.03$ & $0.71 \pm 0.09$ & $0.76 \pm 0.14$ & $0.63 \pm 0.07$ & $0.72 \pm 0.03$ & $0.64 \pm 0.04$ \\
\hline Testis (L) & $0.71 \pm 0.04$ & $0.76 \pm 0.05$ & $0.69 \pm 0.03$ & $0.77 \pm 0.15$ & $0.86 \pm 0.06$ & $0.75 \pm 0.02$ & $0.69 \pm 0.03$ & $0.73 \pm 0.03$ \\
\hline Testis (R) & $0.74 \pm 0.03$ & $0.71 \pm 0.05$ & $0.74 \pm 0.03$ & $0.67 \pm 0.11$ & $0.66 \pm 0.15$ & $0.72 \pm 0.07$ & $0.74 \pm 0.03$ & $0.78 \pm 0.01$ \\
\hline
\end{tabular}

Data are expressed as mean \pm SEM; treatment: G-II to G-IV $=4$ weeks, G-V to $\mathrm{G}-\mathrm{VI}=6$ weeks

${ }^{a} p<0.05$ vs control; one-way ANOVA. L-left, R-right

Table 5 Effects of sub-acute oral administration of BA and APAP on selected biochemical parameters of Wistar rats ( $n=5 /$ sex)

\begin{tabular}{|c|c|c|c|c|c|c|c|c|}
\hline \multirow[t]{2}{*}{ Parameters } & \multirow{2}{*}{$\begin{array}{l}\text { G-I } \\
\text { Control }\end{array}$} & \multirow{2}{*}{$\begin{array}{l}\text { G-II } \\
\text { BA } \\
1 \mathrm{mg} / \mathrm{kg}\end{array}$} & \multirow{2}{*}{$\begin{array}{l}\text { G-III } \\
\text { BA } \\
5 \mathrm{mg} / \mathrm{kg}\end{array}$} & \multirow{2}{*}{$\begin{array}{l}\text { G-IV } \\
\text { BA } \\
10 \mathrm{mg} / \mathrm{kg}\end{array}$} & \multirow{2}{*}{$\begin{array}{l}\text { G-V } \\
\text { APAP } \\
50 \mathrm{mg} / \mathrm{kg}\end{array}$} & \multicolumn{3}{|c|}{ Satellite groups } \\
\hline & & & & & & $\begin{array}{l}\text { G-VI } \\
\text { Control }\end{array}$ & $\begin{array}{l}\text { G-VII } \\
\text { BA } \\
10 \mathrm{mg} / \mathrm{kg}\end{array}$ & $\begin{array}{l}\text { G-VIII } \\
\text { APAP } \\
50 \mathrm{mg} / \mathrm{kg}\end{array}$ \\
\hline \multicolumn{9}{|l|}{ Female } \\
\hline SGOT (U/L) & $84.11 \pm 5.1$ & $92.06 \pm 3.3$ & $108.53 \pm 7.8^{\mathrm{a}}$ & $148.8 \pm 9.6^{b}$ & $195.05 \pm 3.7^{\mathrm{a}}$ & $88.58 \pm 6.8$ & $81.97 \pm 5.7$ & $113.38 \pm 9.1$ \\
\hline SGPT (U/L) & $80.55 \pm 6.3$ & $81.45 \pm 9.9$ & $81.49 \pm 12.6$ & $84.12 \pm 1.4$ & $115.49 \pm 12.3^{a}$ & $84.19 \pm 9.4$ & $81.56 \pm 7.3$ & $67.77 \pm 8.4$ \\
\hline $\operatorname{ALP}(\mathrm{U} / \mathrm{L})$ & $86.67 \pm 1.3$ & $107.07 \pm 3.6^{\mathrm{a}}$ & $110.0 \pm 4.9^{\mathrm{b}}$ & $308.12 \pm 8.3^{\mathrm{a}}$ & $194.40 \pm 5.6^{a}$ & $92.09 \pm 6.4$ & $92.53 \pm 7.0$ & $139.7 \pm 13.8$ \\
\hline Bili (mg/dl) & $0.76 \pm 0.06$ & $0.69 \pm 0.08^{b}$ & $0.69 \pm 0.01^{b}$ & $0.68 \pm 0.0^{b}$ & $0.67 \pm 0.15^{b}$ & $0.72 \pm 0.06$ & $0.74 \pm 0.04$ & $0.85 \pm 0.06$ \\
\hline Alb (gm/dl) & $3.76 \pm 0.08$ & $4.01 \pm 0.40$ & $4.90 \pm 0.22^{\mathrm{a}}$ & $4.90 \pm 0.35^{\mathrm{a}}$ & $4.09 \pm 0.72^{\mathrm{a}}$ & $4.23 \pm 0.32$ & $4.26 \pm 0.40$ & $3.83 \pm 0.57$ \\
\hline Urea (mg/dl) & $21.55 \pm 0.9$ & $24.01 \pm 0.5$ & $24.89 \pm 1.5$ & $39.80 \pm 0.3^{a}$ & $33.40 \pm 4.5^{\mathrm{a}}$ & $28.19 \pm 0.9$ & $25.93 \pm 1.1$ & $28.36 \pm 1.1$ \\
\hline $\mathrm{Crt}(\mathrm{mg} / \mathrm{dl})$ & $0.60 \pm 0.11$ & $0.77 \pm 0.03$ & $0.73 \pm 0.04$ & $0.76 \pm 0.02^{\mathrm{a}}$ & $0.58 \pm 0.08^{\mathrm{a}}$ & $0.64 \pm 0.02$ & $0.67 \pm 0.06$ & $0.56 \pm 0.04$ \\
\hline \multicolumn{9}{|l|}{ Male } \\
\hline SGOT (U/L) & $71.02 \pm 8.09$ & $93.63 \pm 5.05$ & $107.64 \pm 4.3^{\mathrm{a}}$ & $155.98 \pm 7.7^{\mathrm{a}}$ & $200.29 \pm 7.43^{a}$ & $94.32 \pm 7.84$ & $83.37 \pm 5.79$ & $81.05 \pm 8.13$ \\
\hline SGPT (U/L) & $62.10 \pm 0.70$ & $69.94 \pm 8.16$ & $78.37 \pm 14.3^{\mathrm{a}}$ & $78.72 \pm 0.51^{\mathrm{a}}$ & $124.32 \pm 13.9^{\mathrm{a}}$ & $73.87 \pm 13.8$ & $75.69 \pm 11.0$ & $77.40 \pm 4.61$ \\
\hline ALP $(U / L)$ & $88.72 \pm 1.55$ & $105.94 \pm 2.2^{\mathrm{a}}$ & $103.15 \pm 4.9^{\mathrm{a}}$ & $321.8 \pm 7.8^{b}$ & $176.40 \pm 2.73^{\mathrm{a}}$ & $98.27 \pm 5.15$ & $90.17 \pm 7.42$ & $104.1 \pm 17.7$ \\
\hline Bili (mg/dl) & $0.82 \pm 0.06$ & $0.78 \pm 0.08^{\mathrm{a}}$ & $0.76 \pm 0.0^{\mathrm{a}}$ & $0.68 \pm 0.0^{b}$ & $0.42 \pm 0.19^{a}$ & $0.80 \pm 0.07$ & $0.73 \pm 0.05$ & $0.85 \pm 0.04$ \\
\hline Alb $(\mathrm{gm} / \mathrm{dl})$ & $4.02 \pm 0.03$ & $4.18 \pm 0.43$ & $4.36 \pm 0.25$ & $4.88 \pm 0.01^{\mathrm{a}}$ & $5.84 \pm 0.44^{\mathrm{a}}$ & $4.43 \pm 0.39$ & $4.20 \pm 0.42$ & $5.02 \pm 0.70$ \\
\hline Urea (mg/dl) & $24.05 \pm 0.16$ & $23.29 \pm 0.43$ & $25.77 \pm 2.10$ & $40.21 \pm 0.28^{\mathrm{a}}$ & $32.17 \pm 4.57^{\mathrm{a}}$ & $20.55 \pm 1.95$ & $19.00 \pm 4.02$ & $21.31 \pm 3.76$ \\
\hline Crt (mg/dl) & $0.62 \pm 0.10$ & $0.78 \pm 0.04$ & $0.76 \pm 0.03$ & $0.80 \pm 0.08^{\mathrm{a}}$ & $0.78 \pm 0.02^{\mathrm{a}}$ & $0.68 \pm 0.03$ & $0.67 \pm 0.04$ & $0.64 \pm 0.07$ \\
\hline
\end{tabular}


Table 6 Effects of sub-acute oral administration of UA and APAP on selected biochemical parameters of Wistar rats $(n=5 /$ sex)

\begin{tabular}{|c|c|c|c|c|c|c|c|c|}
\hline \multirow[t]{2}{*}{ Parameters } & \multirow{2}{*}{$\begin{array}{l}\text { G-I } \\
\text { Control }\end{array}$} & \multirow{2}{*}{$\begin{array}{l}\text { G-II } \\
\text { UA } \\
1 \mathrm{mg} / \mathrm{kg}\end{array}$} & \multirow{2}{*}{$\begin{array}{l}\text { G-III } \\
\text { UA } \\
5 \mathrm{mg} / \mathrm{kg}\end{array}$} & \multirow{2}{*}{$\begin{array}{l}\text { G-IV } \\
\text { UA } \\
10 \mathrm{mg} / \mathrm{kg}\end{array}$} & \multirow{2}{*}{$\begin{array}{l}\text { G-V } \\
\text { APAP } \\
50 \mathrm{mg} / \mathrm{kg}\end{array}$} & \multicolumn{3}{|c|}{ Satellite groups } \\
\hline & & & & & & $\begin{array}{l}\mathrm{G}-\mathrm{VI} \\
\text { Control }\end{array}$ & $\begin{array}{l}\text { G-VII } \\
\text { UA } \\
10 \mathrm{mg} / \mathrm{kg}\end{array}$ & $\begin{array}{l}\text { G-VIII } \\
\text { APAP } \\
50 \mathrm{mg} / \mathrm{kg}\end{array}$ \\
\hline \multicolumn{9}{|l|}{ Female } \\
\hline SGOT (U/L) & $68.77 \pm 9.03$ & $96.60 \pm 4.2^{a}$ & $111.0 \pm 8.3^{b}$ & $165.7 \pm 0.3^{b}$ & $182.19 \pm 4.4^{\mathrm{a}}$ & $91.59 \pm 7.4$ & $73.68 \pm 5.2$ & $101.04 \pm 2.9$ \\
\hline SGPT (U/L) & $61.32 \pm 6.17$ & $56.80 \pm 10.6$ & $57.7 \pm 14.25$ & $62.68 \pm 2.40$ & $111.40 \pm 11.5^{\mathrm{a}}$ & $90.67 \pm 10.0$ & $83.41 \pm 8.3$ & $68.23 \pm 7.52$ \\
\hline ALP (U/L) & $85.48 \pm 5.40$ & $115.00 \pm 6.2^{\mathrm{a}}$ & $117.7 \pm 8.8^{b}$ & $184.0 \pm 2.7^{b}$ & $193.60 \pm 6.4^{c}$ & $99.64 \pm 7.52$ & $92.04 \pm 6.4$ & $129.57 \pm 8.80$ \\
\hline Bili (mg/dl) & $0.71 \pm 0.07$ & $0.64 \pm 0.05$ & $0.57 \pm 0.05$ & $0.46 \pm 0.00$ & $0.78 \pm 0.04$ & $0.79 \pm 0.04$ & $0.76 \pm 0.05$ & $0.82 \pm 0.06$ \\
\hline Alb (gm/dl) & $3.70 \pm 0.45$ & $3.95 \pm 0.35$ & $4.14 \pm 0.34$ & $3.82 \pm 0.11$ & $4.24 \pm 0.68$ & $4.00 \pm 0.30$ & $4.31 \pm 0.34$ & $3.83 \pm 0.40$ \\
\hline Urea (mg/dl) & $22.51 \pm 1.81$ & $23.24 \pm 1.06$ & $23.73 \pm 2.16$ & $20.22 \pm 0.31$ & $28.13 \pm 5.35$ & $19.82 \pm 0.99$ & $17.28 \pm 0.39$ & $19.25 \pm 1.02$ \\
\hline Crt (mg/dl) & $0.53 \pm 0.14$ & $0.74 \pm 0.02$ & $0.72 \pm 0.04$ & $0.77 \pm 0.01$ & $0.72 \pm 0.10$ & $0.65 \pm 0.06$ & $0.76 \pm 0.04$ & $0.64 \pm 0.03$ \\
\hline \multicolumn{9}{|l|}{ Male } \\
\hline SGOT (U/L) & $84.83 \pm 6.60$ & $104.60 \pm 3.7$ & $108.36 \pm 4.1$ & $197.2 \pm 0.8^{b}$ & $190.13 \pm 4.28^{a}$ & $99.19 \pm 8.45$ & $92.38 \pm 7.58$ & $118.18 \pm 4.34$ \\
\hline SGPT (U/L) & $62.31 \pm 11.4$ & $61.60 \pm 8.35$ & $60.24 \pm 10.7$ & $60.04 \pm 1.47$ & $121.09 \pm 15.8^{\mathrm{a}}$ & $84.06 \pm 12.9$ & $75.7 \pm 11.09$ & $76.08 \pm 2.47$ \\
\hline ALP (U/L) & $73.91 \pm 2.08$ & $103.60 \pm 4.9^{a}$ & $157.0 \pm 7.4^{b}$ & $209.9 \pm 1.9^{b}$ & $182.80 \pm 7.2^{\mathrm{a}}$ & $103.96 \pm 5.6$ & $106.38 \pm 7.5^{c}$ & $102.74 \pm 17.2$ \\
\hline Bili (mg/dl) & $0.75 \pm 0.07$ & $0.75 \pm 0.03$ & $0.71 \pm 0.04$ & $0.66 \pm 0.01$ & $0.77 \pm 0.07$ & $0.87 \pm 0.07$ & $0.79 \pm 0.05$ & $0.84 \pm 0.09$ \\
\hline Alb (gm/dl) & $4.05 \pm 0.66$ & $4.23 \pm 0.32$ & $4.68 \pm 0.56$ & $4.09 \pm 0.07$ & $5.71 \pm 0.36^{c}$ & $4.73 \pm 0.36$ & $1.23 \pm 0.08$ & $4.81 \pm 0.51$ \\
\hline Urea $(\mathrm{mg} / \mathrm{dl})$ & $21.23 \pm 1.07$ & $23.58 \pm 0.84$ & $23.52 \pm 2.04$ & $22.08 \pm 0.23$ & $36.89 \pm 4.82^{c}$ & $22.86 \pm 2.59$ & $23.52 \pm 3.96$ & $21.89 \pm 0.95$ \\
\hline Crt (mg/dl) & $0.70 \pm 0.08$ & $0.75 \pm 0.04$ & $0.76 \pm 0.03$ & $0.80 \pm 0.00$ & $0.81 \pm 0.05$ & $0.71 \pm 0.03$ & $0.72 \pm 0.04$ & $0.73 \pm 0.02$ \\
\hline
\end{tabular}

Data are expressed as mean \pm SEM; treatment: G-II to G-IV $=4$ weeks, $\mathrm{G}-\mathrm{V}$ to $\mathrm{G}-\mathrm{VI}=6$ weeks

${ }^{\mathrm{a}} p<0.05,{ }^{\mathrm{b}} p<0.01$ vs control; ${ }^{\mathrm{c}} p<0.05$ vs satellite control, one-way ANOVA

Table 7 Effects of sub-acute oral administration of BA and APAP on selected hematological parameters of Wistar rats ( $n=5 /$ sex)

\begin{tabular}{|c|c|c|c|c|c|c|c|c|}
\hline \multirow[t]{2}{*}{ Parameters } & \multirow{2}{*}{$\begin{array}{l}\text { G-I } \\
\text { Control }\end{array}$} & \multirow{2}{*}{$\begin{array}{l}\mathrm{G}-\mathrm{II} \\
\mathrm{BA} \\
1 \mathrm{mg} / \mathrm{kg}\end{array}$} & \multirow{2}{*}{$\begin{array}{l}\mathrm{G}-\mathrm{III} \\
\mathrm{BA} \\
5 \mathrm{mg} / \mathrm{kg}\end{array}$} & \multirow{2}{*}{$\begin{array}{l}\text { G-IV } \\
\text { BA } \\
10 \mathrm{mg} / \mathrm{kg}\end{array}$} & \multirow{2}{*}{$\begin{array}{l}\text { G-V } \\
\text { APAP } \\
50 \mathrm{mg} / \mathrm{kg}\end{array}$} & \multicolumn{3}{|c|}{ Satellite groups } \\
\hline & & & & & & $\begin{array}{l}\text { G-VI } \\
\text { Control }\end{array}$ & $\begin{array}{l}\text { G-VII } \\
\text { BA } \\
10 \mathrm{mg} / \mathrm{kg}\end{array}$ & $\begin{array}{l}\text { G-VIII } \\
\text { APAP } \\
50 \mathrm{mg} / \mathrm{kg}\end{array}$ \\
\hline \multicolumn{9}{|l|}{ Female } \\
\hline $\mathrm{RBC}$ & $7.30 \pm 0.24$ & $7.82 \pm 0.21$ & $7.64 \pm 0.12$ & $7.24 \pm 0.17$ & $3.21 \pm 0.23^{a}$ & $7.58 \pm 0.29$ & $7.89 \pm 0.23$ & $7.45 \pm 0.21$ \\
\hline WBC & $7280 \pm 386.5$ & $7726 \pm 476.3$ & $7100 \pm 104.8$ & $7220 \pm 0.17$ & $3401 \pm 226.6^{\mathrm{a}}$ & $7560 \pm 213.6$ & $7441 \pm 250.2$ & $7006 \pm 107.8$ \\
\hline Neut (\%) & $21.60 \pm 3.50$ & $21.80 \pm 0.37$ & $23.80 \pm 0.37$ & $23.80 \pm 1.59$ & $41.80 \pm 2.6^{\mathrm{a}}$ & $22.00 \pm 2.44$ & $21.00 \pm 1.58$ & $19.40 \pm 0.67$ \\
\hline Lym (\%) & $36.20 \pm 10.5$ & $38.60 \pm 11.6$ & $40.40 \pm 9.92$ & $62.40 \pm 5.9^{b}$ & $29.80 \pm 4.6^{a}$ & $36.00 \pm 2.34$ & $38.00 \pm 1.76$ & $38.60 \pm 2.42$ \\
\hline Eos (\%) & $2.80 \pm 1.11$ & $2.20 \pm 0.58$ & $2.80 \pm 0.66$ & $5.20 \pm 0.58^{b}$ & $2.00 \pm 0.44$ & $2.20 \pm 0.37$ & $2.00 \pm 0.44$ & $1.60 \pm 0.40$ \\
\hline Mono (\%) & $2.20 \pm 1.23$ & $2.80 \pm 0.58$ & $2.20 \pm 0.58$ & $1.80 \pm 0.37^{a}$ & $1.40 \pm 0.24^{\mathrm{a}}$ & $2.60 \pm 0.24$ & $2.20 \pm 0.20$ & $1.40 \pm 0.24$ \\
\hline PCV (\%) & $32.41 \pm 3.43$ & $40.17 \pm 3.34$ & $42.64 \pm 1.4^{\mathrm{a}}$ & $42.21 \pm 2.1^{\mathrm{a}}$ & $29.91 \pm 1.5^{\mathrm{a}}$ & $32.86 \pm 1.76$ & $40.49 \pm 1.50^{c}$ & $39.67 \pm 0.88$ \\
\hline $\mathrm{Hb}(\mathrm{gm} / \mathrm{dL})$ & $12.98 \pm 0.47$ & $12.69 \pm 0.35$ & $12.56 \pm 0.25$ & $12.60 \pm 0.27$ & $9.04 \pm 0.37^{a}$ & $12.47 \pm 0.34$ & $12.99 \pm 0.39$ & $12.15 \pm 0.17$ \\
\hline $\mathrm{PI}\left(\times 10^{2} / \mathrm{cmm}\right)$ & $1863 \pm 21.25$ & $1774 \pm 43.63$ & $1827 \pm 20.21$ & $1783 \pm 16.95$ & $1263 \pm 30.9^{\mathrm{a}}$ & $1804 \pm 30.43$ & $1747 \pm 27.08$ & $1632 \pm 27.49$ \\
\hline \multicolumn{9}{|l|}{ Male } \\
\hline $\mathrm{RBC}$ & $8.20 \pm 0.15$ & $8.65 \pm 0.38$ & $8.11 \pm 0.18$ & $7.73 \pm 0.20$ & $3.64 \pm 0.13^{\mathrm{a}}$ & $8.11 \pm 0.18$ & $8.13 \pm 0.25$ & $8.01 \pm 0.10$ \\
\hline WBC & $8540 \pm 321.8$ & $8900 \pm 130.3$ & $7640 \pm 143.5$ & $8078 \pm 162.4$ & $5001 \pm 298.2^{\mathrm{a}}$ & $8012 \pm 355.4$ & $8122 \pm 101.08$ & $7948 \pm 96.8$ \\
\hline Neut (\%) & $29.60 \pm 1.5$ & $30.40 \pm 0.67$ & $31.00 \pm 0.54$ & $34.80 \pm 1.77^{\mathrm{a}}$ & $43.20 \pm 2.47^{a}$ & $29.40 \pm 1.36$ & $23.20 \pm 1.70$ & $20.60 \pm 0.81$ \\
\hline Lym (\%) & $32.00 \pm 10.15$ & $38.80 \pm 10.24$ & $42.20 \pm 6.81^{\mathrm{a}}$ & $60.00 \pm 2.09^{b}$ & $43.20 \pm 6.46^{\mathrm{a}}$ & $72.00 \pm 2.70$ & $72.20 \pm 2.15$ & $63.60 \pm 1.56$ \\
\hline Eos (\%) & $2.80 \pm 0.37$ & $2.40 \pm 0.87$ & $5.80 \pm 0.82^{b}$ & $7.60 \pm 0.50^{b}$ & $1.60 \pm 0.40$ & $2.20 \pm 0.37$ & $2.00 \pm 0.31$ & $1.80 \pm 0.37$ \\
\hline Mono (\%) & $2.00 \pm 1.00$ & $2.00 \pm 0.44$ & $2.40 \pm 0.24$ & $2.00 \pm 0.54$ & $1.40 \pm 0.24^{\mathrm{a}}$ & $1.60 \pm 0.40$ & $1.40 \pm 0.24$ & $1.60 \pm 0.40$ \\
\hline PCV (\%) & $39.19 \pm 6.06$ & $44.22 \pm 1.48$ & $47.28 \pm 0.58$ & $48.04 \pm 2.24$ & $30.92 \pm 0.84$ & $40.14 \pm 3.19$ & $45.10 \pm 2.15$ & $43.13 \pm 1.19$ \\
\hline $\mathrm{Hb}(\mathrm{gm} / \mathrm{dL})$ & $13.68 \pm 0.31$ & $12.55 \pm 0.33$ & $13.62 \pm 0.04$ & $13.58 \pm 0.10$ & $9.21 \pm 0.13^{\mathrm{a}}$ & $13.36 \pm 0.31$ & $13.04 \pm 0.32$ & $12.46 \pm 0.27$ \\
\hline $\mathrm{PI}\left(\times 10^{2} / \mathrm{cmm}\right)$ & $1810 \pm 33.50$ & $1821 \pm 17.24$ & $1826 \pm 18.97$ & $1822 \pm 20.10$ & $1306 \pm 12.47^{\mathrm{a}}$ & $1811 \pm 13.24$ & $1803 \pm 10.96$ & $1695 \pm 43.14$ \\
\hline
\end{tabular}




\section{Results}

\section{Limit test}

Administration of a single oral dose of $2000 \mathrm{mg} / \mathrm{kg}$ of $\mathrm{BA}$ and UA to mice did not cause any mortality during the 2-week observation period. Therefore, it can be concluded that the LD50 for both the test agents was found to be $>2000 \mathrm{mg} / \mathrm{kg}$ in mice.

\section{Sub-acute toxicity study \\ Effect on body weights}

Oral administration of BA (Table 1) and UA (Table 2) did not reveal any significant changes in body weights of animals from both the sexes in all the groups; also, no mortality was observed during the study.

\section{Effects on ROW}

Oral administration of BA (Table 3) and UA (Table 4) did not reveal any noticeable changes in their ROWs in both female and male animals of all the groups. However, animals administered APAP showed an increase in the ROW of the spleen.

\section{Effects on biochemical parameters}

The sub-acute oral administration of BA revealed a dose-dependent increase in SGOT (serum glutamic oxaloacetic transaminase) and ALP (alkaline phosphatase) in animals of both the sexes, and the highest treated dose $(10 \mathrm{mg} / \mathrm{kg})$ showed an elevation in urea levels (Table 5). Sub-acute oral administration of UA revealed elevations in the levels of SGOT and ALP in a dose-dependent manner, whereas increased urea levels were observed in only the highest treated dose $(10 \mathrm{mg} / \mathrm{kg})$ (Table 6$)$. All these parameters were recoverable once dosing was discontinued as evidenced by the values of the satellite group (Tables 5 and 6). Animals treated with APAP showed an increase in SGOT, SGPT (serum glutamic pyruvic transaminase), ALP, and urea values which were however recoverable after the dosing was discontinued (Tables 5 and 6).

\section{Effects on hematological parameters}

The oral administration of $\mathrm{BA}$ revealed a dosedependent increase in lymphocyte count in all the

Table 8 Effects of sub-acute oral administration of UA and APAP on selected hematological parameters of Wistar rats $(n=5 /$ sex)

\begin{tabular}{|c|c|c|c|c|c|c|c|c|}
\hline \multirow[t]{2}{*}{ Parameters } & \multirow{2}{*}{$\begin{array}{l}\text { G-I } \\
\text { Control }\end{array}$} & \multirow{2}{*}{$\begin{array}{l}\text { G-II } \\
\text { UA } \\
1 \mathrm{mg} / \mathrm{kg}\end{array}$} & \multirow{2}{*}{$\begin{array}{l}\text { G-III } \\
\text { UA } \\
5 \mathrm{mg} / \mathrm{kg}\end{array}$} & \multirow{2}{*}{$\begin{array}{l}\text { G-IV } \\
\text { UA } \\
10 \mathrm{mg} / \mathrm{kg}\end{array}$} & \multirow{2}{*}{$\begin{array}{l}\text { G-V } \\
\text { APAP } \\
50 \mathrm{mg} / \mathrm{kg}\end{array}$} & \multicolumn{3}{|c|}{ Satellite groups } \\
\hline & & & & & & $\begin{array}{l}\text { G-VI } \\
\text { Control }\end{array}$ & $\begin{array}{l}\text { G-VII } \\
\text { UA } \\
10 \mathrm{mg} / \mathrm{kg}\end{array}$ & $\begin{array}{l}\text { G-VIII } \\
\text { APAP } \\
50 \mathrm{mg} / \mathrm{kg}\end{array}$ \\
\hline \multicolumn{9}{|l|}{ Female } \\
\hline $\mathrm{RBC}(/ \mathrm{cmm})$ & $7.51 \pm 0.19$ & $7.16 \pm 0.10$ & $7.51 \pm 0.20$ & $7.11 \pm 0.28$ & $3.42 \pm 0.25^{\mathrm{a}}$ & $7.79 \pm 0.17$ & $7.49 \pm 0.19$ & $7.64 \pm 0.20$ \\
\hline WBC (/cmm) & $7480 \pm 285.3$ & $7253 \pm 266.6$ & $7529 \pm 232.9$ & $3846 \pm 227.4^{a}$ & $3811 \pm 137.4^{a}$ & $7784 \pm 186.3$ & $7645 \pm 220.5$ & $7269 \pm 192.3$ \\
\hline Neut (\%) & $21.00 \pm 3.56$ & $19.20 \pm 0.58$ & $19.40 \pm 0.74$ & $41.80 \pm 8.32^{a}$ & $19.40 \pm 0.92^{a}$ & $21.80 \pm 1.46$ & $18.60 \pm 0.50$ & $20.80 \pm 1.15$ \\
\hline Lym (\%) & $68.00 \pm 3.53$ & $65.00 \pm 2.07$ & $54.20 \pm 1.93$ & $25.20 \pm 4.90^{\mathrm{a}}$ & $79.60 \pm 5.95^{c}$ & $66.80 \pm 2.98$ & $70.40 \pm 3.00$ & $62.20 \pm 5.07$ \\
\hline Eos (\%) & $4.60 \pm 0.24$ & $4.60 \pm 0.24$ & $3.60 \pm 0.50$ & $1.40 \pm 0.24^{\mathrm{a}}$ & $1.20 \pm 0.20^{b}$ & $4.40 \pm 0.70$ & $3.80 \pm 0.37$ & $4.20 \pm 0.58$ \\
\hline Mono (\%) & $1.40 \pm 0.24$ & $1.20 \pm 0.20$ & $1.20 \pm 0.20$ & $1.40 \pm 0.24$ & $1.40 \pm 0.24$ & $1.60 \pm 0.40$ & $1.00 \pm 0.00$ & $1.60 \pm 0.40$ \\
\hline PCV (\%) & $34.60 \pm 2.04$ & $39.46 \pm 0.60$ & $36.19 \pm 1.48$ & $25.14 \pm 1.68^{\mathrm{a}}$ & $24.48 \pm 3.89^{c}$ & $42.94 \pm 1.94$ & $34.02 \pm 1.46$ & $31.90 \pm 3.63$ \\
\hline $\mathrm{Hb}(\mathrm{gm} / \mathrm{dL})$ & $11.77 \pm 0.56$ & $11.87 \pm 0.20$ & $11.91 \pm 0.22$ & $6.42 \pm 0.21^{b}$ & $6.79 \pm 0.38^{b}$ & $11.82 \pm 0.19$ & $12.01 \pm 0.19$ & $12.01 \pm 0.24$ \\
\hline $\mathrm{PI}\left(\times 10^{2} / \mathrm{cmm}\right)$ & $1863 \pm 21.25$ & $1776 \pm 13.8$ & $1775 \pm 34.7$ & $1215 \pm 31.84^{b}$ & $1280 \pm 86.89^{a}$ & $1775 \pm 42.18$ & $1760 \pm 38.16$ & $1755 \pm 91.99$ \\
\hline \multicolumn{9}{|l|}{ Male } \\
\hline $\mathrm{RBC}(/ \mathrm{cmm})$ & $7.67 \pm 0.20$ & $7.78 \pm 0.26$ & $7.77 \pm 0.21$ & $7.99 \pm 0.10$ & $3.37 \pm 0.12^{b}$ & $8.00 \pm 0.06$ & $7.84 \pm 0.16$ & $8.14 \pm 0.10$ \\
\hline WBC (/cmm) & $8280 \pm 491.3$ & $8010 \pm 83.5$ & $7573 \pm 225.5$ & $4778 \pm 276.6^{a}$ & $4442 \pm 254.6^{a}$ & $7943 \pm 321.1$ & $8590 \pm 313.5$ & $8136 \pm 167.1$ \\
\hline Neutr (\%) & $21.60 \pm 1.50$ & $19.60 \pm 0.67$ & $19.40 \pm 0.50$ & $41.40 \pm 1.12^{\mathrm{a}}$ & $36.80 \pm 4.80^{b}$ & $26.80 \pm 2.17$ & $24.60 \pm 1.20$ & $22.20 \pm 1.82$ \\
\hline Lym (\%) & $66.60 \pm 6.16$ & $72.40 \pm 2.24$ & $74.20 \pm 1.24$ & $38.20 \pm 2.59^{a}$ & $61.80 \pm 6.87$ & $80.80 \pm 4.09$ & $77.40 \pm 3.68$ & $63.00 \pm 5.70$ \\
\hline Eos (\%) & $3.40 \pm 0.67$ & $3.20 \pm 0.37$ & $4.00 \pm 0.70$ & $4.20 \pm 0.37$ & $1.40 \pm 0.24$ & $4.40 \pm 0.24$ & $2.00 \pm 0.44$ & $3.00 \pm 0.70$ \\
\hline Mono (\%) & $1.20 \pm 0.20$ & $1.60 \pm 0.40$ & $1.20 \pm 0.20$ & $1.40 \pm 0.24$ & $1.40 \pm 0.40$ & $1.20 \pm 0.20$ & $1.60 \pm 0.40$ & $1.20 \pm 0.20$ \\
\hline PCV (\%) & $45.68 \pm 3.21$ & $40.18 \pm 1.12$ & $40.19 \pm 0.54$ & $32.04 \pm 1.32$ & $26.15 \pm 1.02^{b}$ & $37.85 \pm 2.72$ & $44.13 \pm 1.77$ & $40.63 \pm 1.31$ \\
\hline $\mathrm{Hb}(\mathrm{gm} / \mathrm{dL})$ & $12.65 \pm 0.46$ & $11.87 \pm 0.33$ & $12.51 \pm 0.15$ & $12.43 \pm 0.23$ & $8.75 \pm 0.48^{c}$ & $12.13 \pm 0.18$ & $12.26 \pm 0.09$ & $11.96 \pm 0.28$ \\
\hline $\mathrm{PI}\left(\times 10^{2} / \mathrm{cmm}\right)$ & $1810 \pm 33.50$ & $1780 \pm 11.05$ & $1805 \pm 5.73$ & $1206 \pm 12.03^{a}$ & $1225 \pm 61.12^{a}$ & $1900 \pm 45.91$ & $1813 \pm 8.32$ & $1763 \pm 57.31$ \\
\hline
\end{tabular}

Data are expressed as mean \pm SEM; treatment: G-II to G-IV $=4$ weeks, G-V to G-VI $=6$ weeks

${ }^{\mathrm{a}} p<0.05$, vs control; ${ }^{\mathrm{b}} p<0.05$, vs control; one-way ANOVA 
treated groups of both the sexes and only the highest treated dose $(10 \mathrm{mg} / \mathrm{kg})$ showed elevated eosinophil count (Table 7). Animals administered with the highest dose $(10 \mathrm{mg} / \mathrm{kg})$ of UA revealed elevated neutrophil count whereas white blood cells (WBC), lymphocyte, hemoglobin $(\mathrm{Hb})$, and platelet $(\mathrm{Pl})$ counts were found to be low. The eosinophil count in male animals was normal whereas female animals treated with the highest dose showed decreased eosinophil count (Table 8). All the abnormal values were recoverable after dosing was discontinued as is evident in the satellite groups (Tables 7 and 8).

\section{Histopathology}

The liver sections of control animals showed a normal architecture (Fig. 1a) whereas animals treated with $10 \mathrm{mg} / \mathrm{kg}$ BA showed leucocytic infiltration, sinusoidal congestion, and aggregation of inflammatory cells in portal triads (Fig. 1b). Similarly, histology of liver in animals treated with $10 \mathrm{mg} / \mathrm{kg}$ UA revealed abnormalities in the form of leucocytic infiltration, sinusoidal congestion, aggregation of inflammatory cells in portal triads, and sinusoidal dilatations (Fig. 1c). The liver tissues of animals treated with APAP revealed several histopathological alterations, such as dilation of central vein indicating backflow of circulation, leucocytic infiltration, sinusoidal congestions, and nuclear pyknosis (Fig. 1d). Kidney sections of control animals showed normal architecture (Fig. 2a). Similarly, histological kidney sections from animals treated with $10 \mathrm{mg} / \mathrm{kg}$ BA did not show any alterations in the architecture of the kidney (Fig. 2b). However, kidney sections from animals treated with $10 \mathrm{mg} / \mathrm{kg}$ UA showed distortion in renal tubules, a decrease in the glomeruli size, and a simultaneous increase in the capsular space (Fig. 2c). Likewise, administration of APAP at $50 \mathrm{mg} / \mathrm{kg}$ showed significant alterations in the treated animals, in the form of dilatation of sub-capsular space of glomeruli, reduction in the glomeruli size, multiple focal tubulo-nephritis, formation of vacuoles, and distorted renal tubules (Fig. 2d). Histology of the spleen of control group
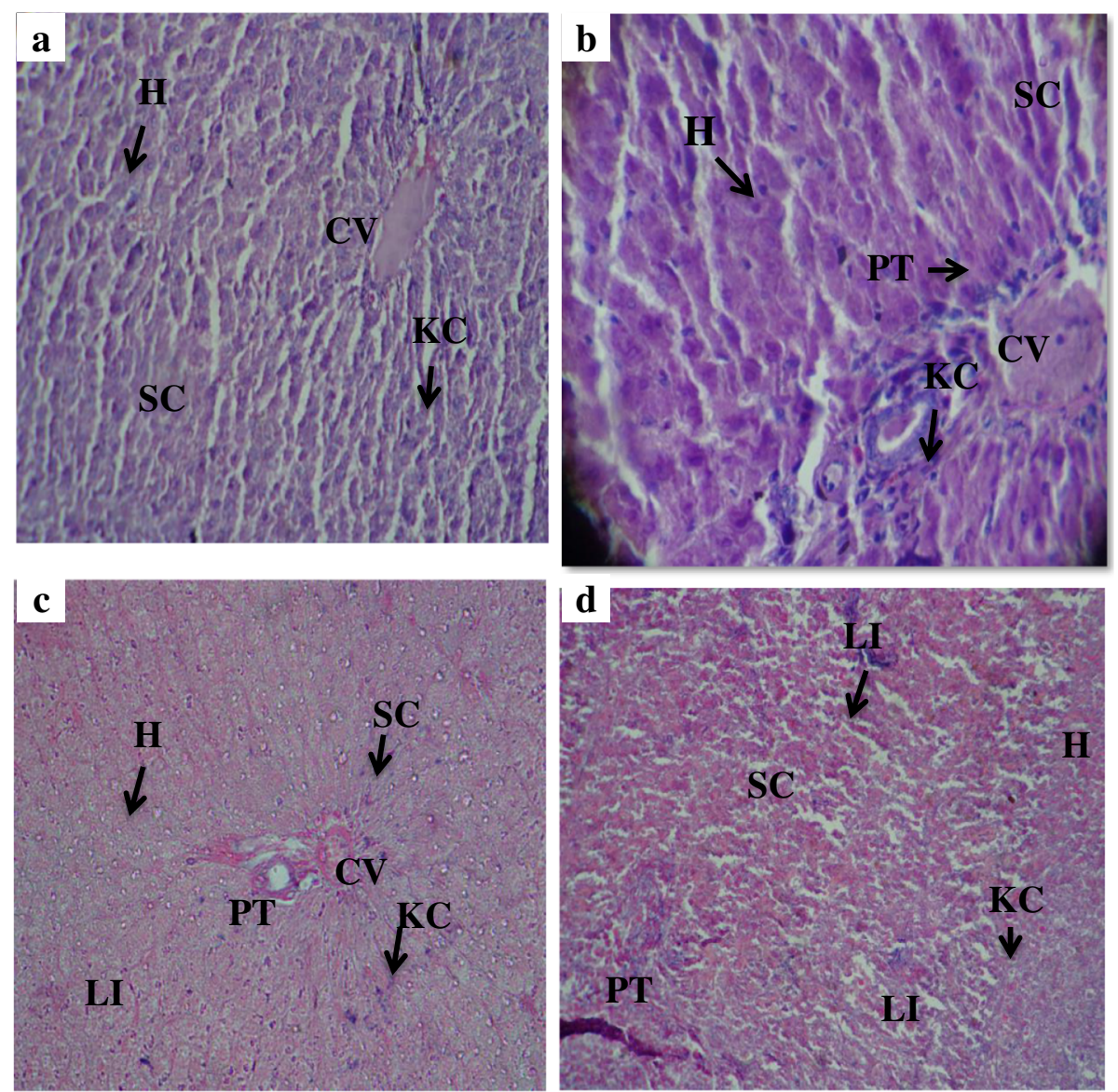

Fig. 1 Liver histological sections of rats in sub-acute toxicity study. a Normal control group; b $10 \mathrm{mg} / \mathrm{kg}$ BA-treated group; c $10 \mathrm{mg} / \mathrm{kg} \cup \mathrm{A}$-treated group; $\mathbf{d} 50 \mathrm{mg} / \mathrm{kg}$ APAP-treated group ( $\times 25$, H\&E stained). CV—central vein, PT—portal triard, SC—-sinusoidal congestion, H—histiocytes, KC-Kupffer cell, LI-leucocytic infiltration 

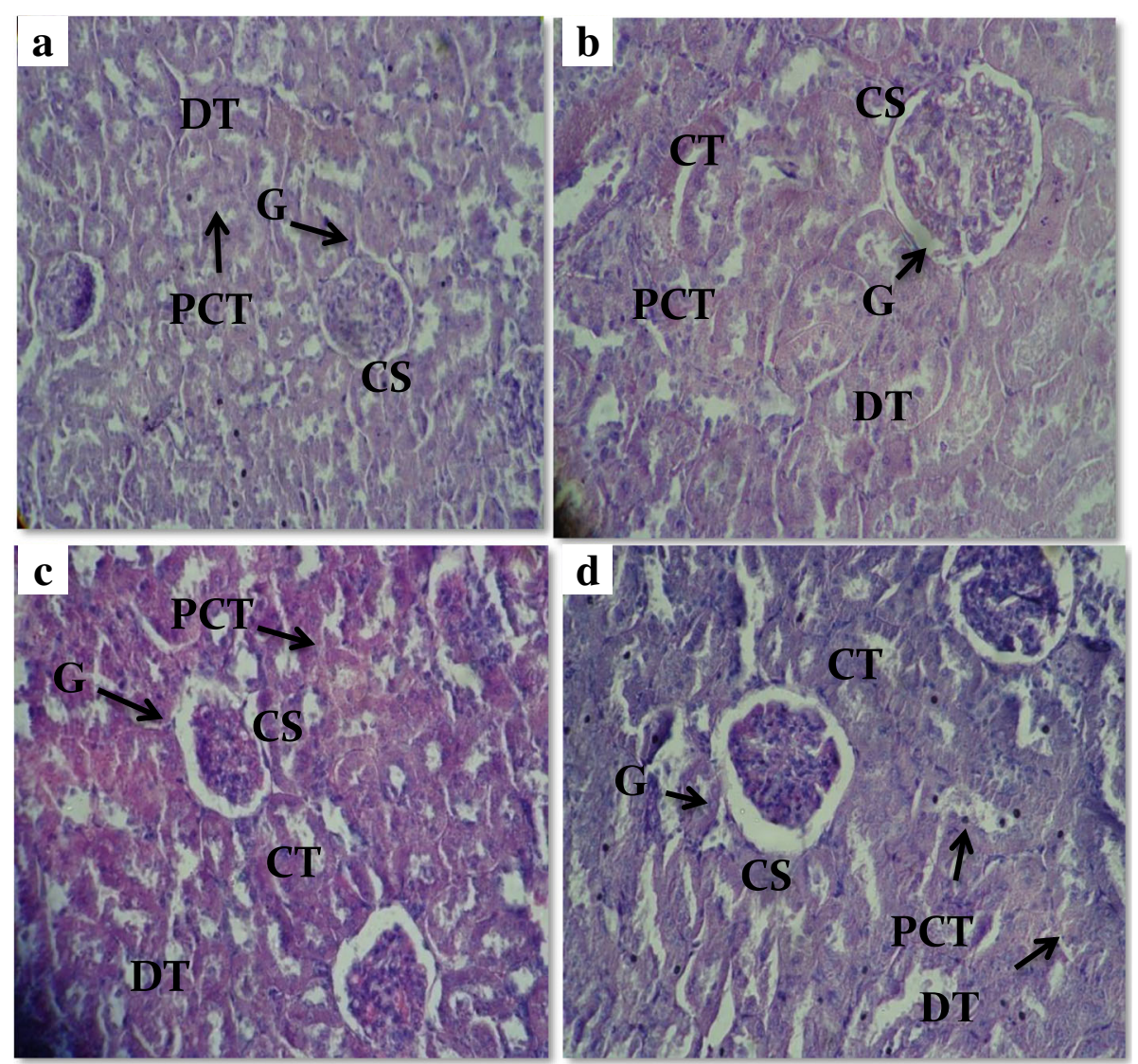

Fig. 2 Kidney histological sections of rats in sub-acute toxicity study. a Normal control group; b 10 mg/kg BA-treated group; c 10 mg/kg UAtreated group; d 50 mg/kg APAP-treated group (×40, H\&E stained). G_-glomeruli, DT_-distal tubules, CS—capsular space, CT-collecting tubules, PCT-proximal convoluted tubule

showed normal architecture (Fig. 3a). However, the spleen of animals treated with the highest test dose of BA (10 $\mathrm{mg} / \mathrm{kg}$ b.w) displayed significant changes in the form of enlarged sinusoids, distorted red pulp area, and neutrophil infiltration (Fig. 3b). On the other hand, animals treated with the highest dose of UA $(10 \mathrm{mg} / \mathrm{kg})$ showed abnormal architecture in the spleen tissues in the form of enlarged sinusoids, distorted red pulp area, and neutrophil infiltration (Fig. 3c). Rats treated with $50 \mathrm{mg} / \mathrm{kg}$ APAP also showed distortions of follicular cells in pulp areas, vacuole formation, and hypercellularity in the red pulp area of the spleen tissue (Fig. 3d). Histology of the heart in control animals revealed normal architecture of the cardiac muscles, connective tissues, and myosin filaments (Fig. 4a). Also, animals administered BA (Fig. 4b), UA (Fig. 4c), and APAP (Fig. 4d) did not reveal any alterations in the histology of the heart tissue.

\section{Discussion}

All chemical constituents and established drugs are evaluated for their acute and repeated 28-day studies to establish their safety profile before their clinical trials and approval into mainstream pharmacology [35]. The present study revealed that the administration of BA and UA did not produce any lasting signs of toxicity or mortality in the experimental animals at $2000 \mathrm{mg} / \mathrm{kg}$ b.w. in mice. All the experimental animals were found to be healthy and normal in their behavior, breathing, posture, and food and water consumption during the experimental period and thereafter. Therefore, the oral LD50 values of both the tested compounds are established to be $>2000 \mathrm{mg} / \mathrm{kg}$ b.w. in mice. A similar study by Lu et al. (2009) also revealed that the LD50 of UA was found to be $9.26 \mathrm{~g} / \mathrm{kg}$ b.w. in mice [36].

Toxicity studies are performed taking a minimum of three doses, viz. low, medium, and high, in the experimental animals, and the effects are compared with positive and negative control animals [37]. The dose which exhibits the most effects during in vivo study is usually selected as the highest dose for toxicity and is considered sufficient to identify the target organ toxicity and optimum therapeutic level is devised [38]. Hence, three doses from each test 

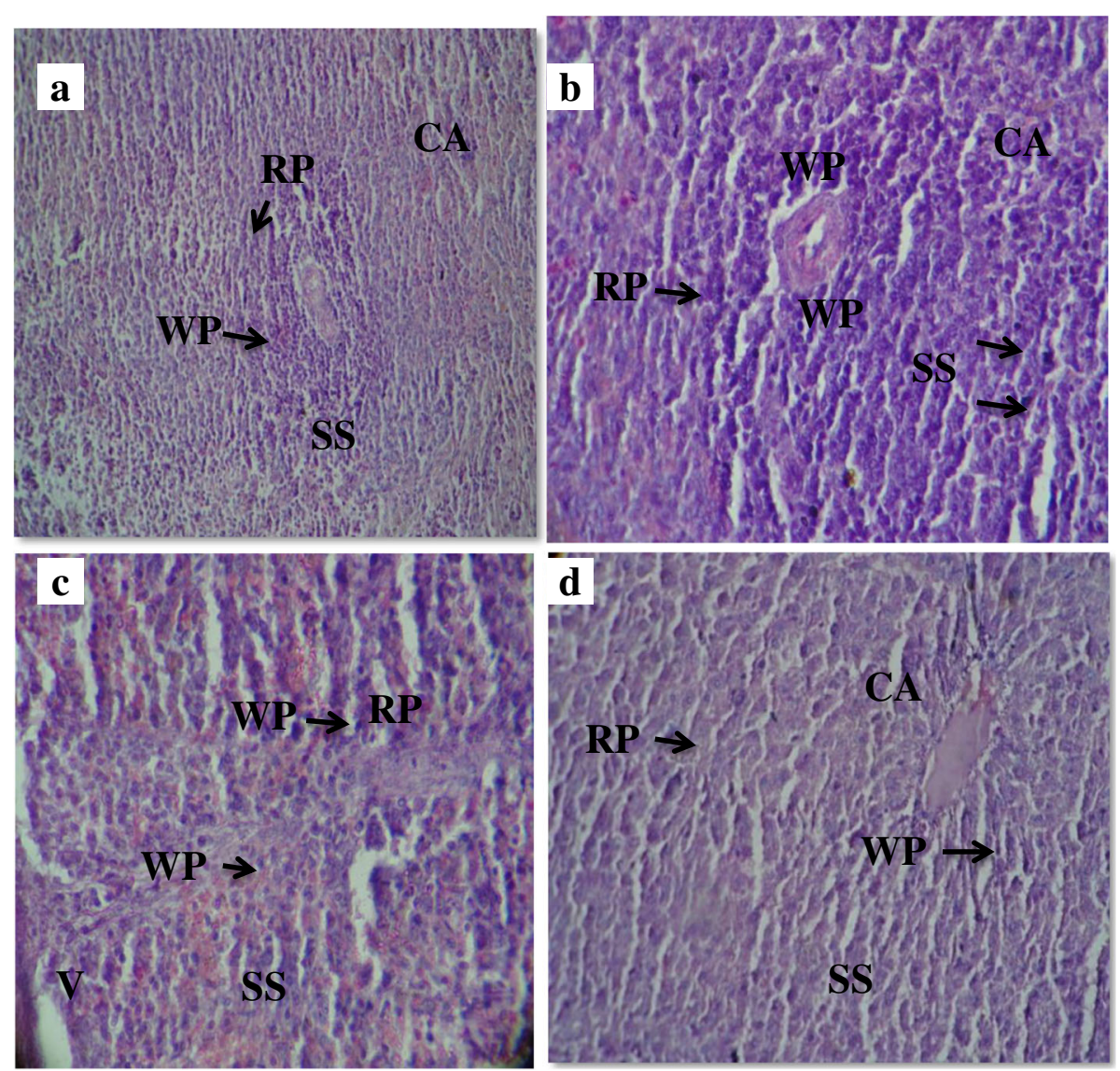

Fig. 3 Spleen histological sections of rats in sub-acute toxicity study. a Normal control group; b 10 mg/kg BA-treated group; c 10 mg/kg UAtreated group; $\mathbf{d} 50$ mg/kg APAP-treated group ( $\times 25$, H\&E stained). WP—white pulp, RP—red pulp, SS—splenic septa, CA—central artery, $\mathrm{V}$ - vacuolization

compound were selected based on in vivo studies. This study did not reveal any abnormalities in body weights, food and water consumption, and ROW in animals administered with BA and UA, and no mortality was observed. Alterations in values of serum enzymes are indicative of hepatotoxicity caused by the administration of any foreign substance [39]. The present study showed an increase in SGOT and ALP following administration of BA and UA indicating hepatocellular damage and hepatotoxicity. Also, animals administered BA and UA revealed an increase in the levels of urea suggesting mild renal toxicity. Urea is a major by-product eliminated by the kidney, and any damage to the kidneys will result in increased urea levels [40]. However, all these effects were reversible as observed in the satellite group. In contrast, a similar study by Mullauer et al. (2011) reported a completed absence of systemic toxicity by BA administered to mice up to a tested dose of 500 $\mathrm{mg} / \mathrm{kg}$ b.w [20].. The hematopoietic system is one of the most sensitive targets for toxic compounds and assists in determining the toxic effects of the extract on the animal's physiological and pathological state [41]. Oral administration of UA revealed a reduction in WBC, lymphocyte, eosinophil, and platelet counts. Similar decrease in platelet count was observed in an acute and sub-acute study on safranal where animals were administered $0.10,0.25$, and $0.50 \mathrm{ml} / \mathrm{kg}$ of safranal [42]. Animals administered BA revealed an increase in lymphocyte and eosinophil counts only. Although animals administered BA and UA revealed mild toxicity as observed by fluctuations in these hematological parameters, these changes were however reversible as evident in the satellite group.

The liver, kidney, spleen, and heart are primary organs affected by metabolic reactions caused by the consumption of toxic compounds [43], and hence, histopathological studies of these tissues were conducted. Animals administered BA and UA caused mild abnormalities in the liver, kidney, and spleen tissues indicating that these compounds possess toxic potentials. The heart tissue however did not show any abnormalities in animals treated with BA and UA. Similar results were reported by Wang et al. (2019) 

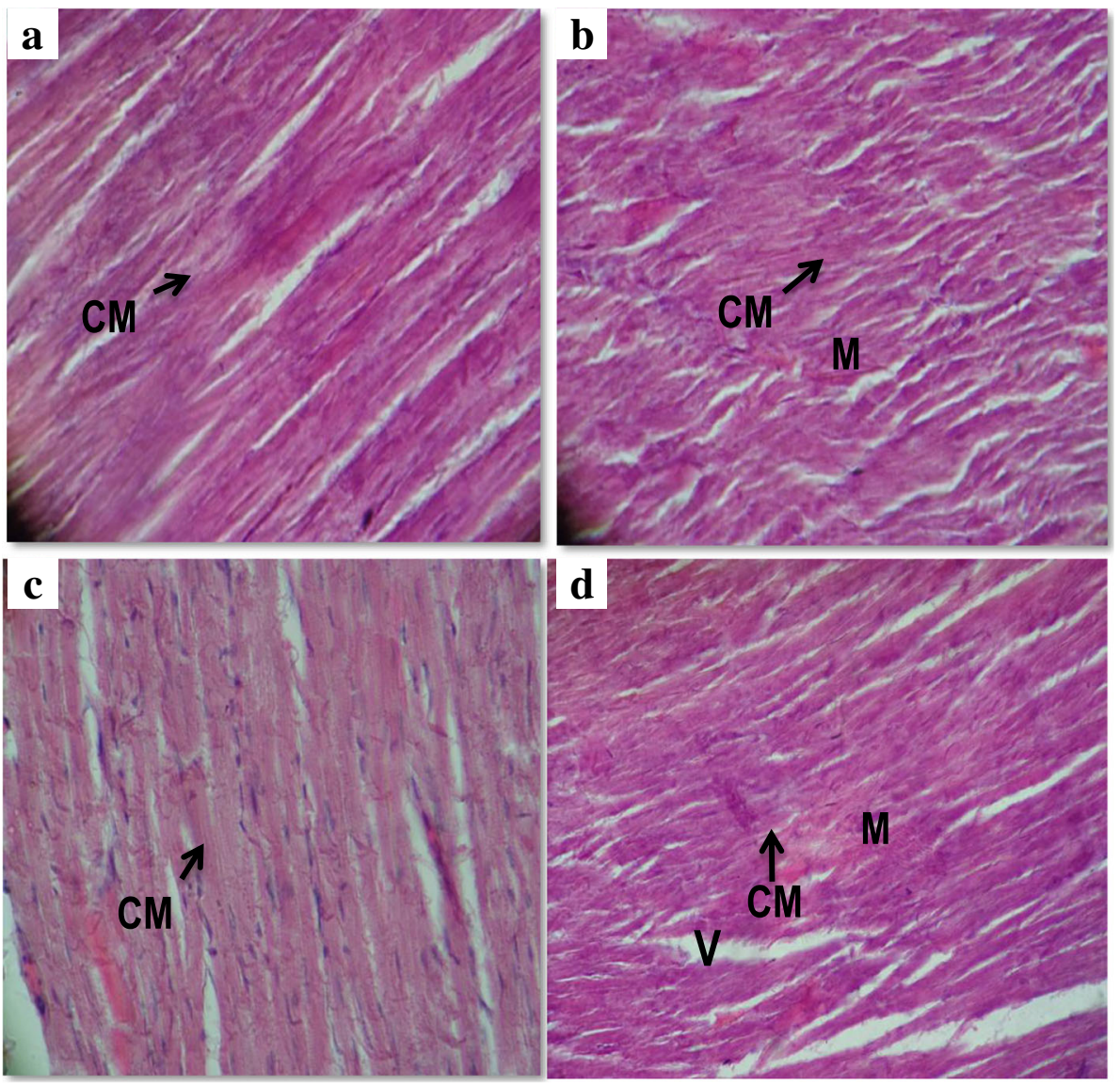

Fig. 4 Heart histological sections of rats in sub-acute toxicity study. a Normal control group; b $10 \mathrm{mg} / \mathrm{kg}$ BA-treated group; c $10 \mathrm{mg} / \mathrm{kg}$ UAtreated group; d $50 \mathrm{mg} / \mathrm{kg}$ APAP-treated group ( $\times 25$, H\&E stained). CM—cardiac muscle, M-myocytes, $\mathrm{V}$ — vacuoles

on acute and sub-acute toxicity assessment of oxyclozanide where histopathological study revealed glomerulonephritis and granular degeneration of tubular epithelium in the kidney tissue and steatosis and granular degeneration in the liver tissue of rats [44].

\section{Conclusion}

The hematological, biochemical, and histopathological studies indicate that the repeated 28-day oral administration of BA and UA caused mild toxic effects which were however reversible on their discontinuation. Further studies to determine the optimum effective and safe dose with minimal or no toxic effects needs to be carried out.

\section{Abbreviations}

APAP: Acetaminophen; Alb: Albumin; ALP: Alkaline phosphatase; ANOVA: Analysis of variance; b.w.: Body weight; BA: Betulinic acid; Bili: Bilirubin; Crt: Creatinine; DMSO: Dimethyl sulphoxide; Eos: Eosinophil; Hb: Hemoglobin; IEC: Institutional Ethics Committee; i.p: Intra peritoneal; Lym: Lymphocyte; Mono: Monocyte; Neut: Neutrophil; OECD: Organization for Economic Cooperation and Development; PBS: Phosphate-buffered saline; PCV: Packed cell volume; PI: Platelet; RBC: Red blood cells; ROW: Relative organ weight; s.c: Subcutaneous; SEM: Standard error of mean;
SGOT: Serum glutamic oxaloacetic transaminase; SGPT: Serum glutamic pyruvic transaminase; UA: Ursolic acid; WBC: White blood cells

\section{Acknowledgements}

The authors wish to thank the Head of the Department of Zoology for providing the necessary facilities to carry out the study. Also, a meritorious fellowship awarded to VM from the UGC, New Delhi, is duly acknowledged.

\section{Authors' contributions}

VM carried out the experiments, analyzed data, and edited the first draft. ADS assisted in carrying out the experiments and analyzing data and wrote the first draft. AKY conceptualized and supervised the study, edited the first draft, and approved it. The authors read and approved the final draft.

\section{Funding}

The study did not receive any funding from agencies in the public, commercial, or not-for-profit sectors.

\section{Availability of data and materials}

All data generated or analyzed during this study are included in this published article (and its supplementary information files).

\section{Ethics approval and consent to participate}

Experiments on animals were approved by the Institutional Ethics Committee (Animal models) of NEHU, Shillong (Vide, Member Secretary, IEC, NEHU, dated December 4, 2014, approval number 01) and all the procedures performed were part of the routine care. All experiments on animals comply with ARRIVE guidelines and have been carried out in accordance with the U.K. Animals (Scientific Procedures) Act, 1986 and associated guidelines (EU 
Directive 2010/63/EU for animal experiments). Also, the animal experiments complied with the ARRIVE guidelines. All the animals were procured from Pasteur Institute, Polo, Shillong, Meghalaya. Pasteur Institute was made aware and consented to the use of animals for this study.

\section{Consent for publication}

Not applicable

\section{Competing interests}

The authors declare that they have no competing interests.

\section{Author details}

'Department of Zoology, North-Eastern Hill University, Shillong, Meghalaya 793022, India. ${ }^{2}$ Present affiliation: Department of Zoology, B. Borooah College, Guwahati, Assam 781007, India.

Received: 26 September 2020 Accepted: 28 December 2020 Published online: 10 February 2021

\section{References}

1. Newman DJ, Cragg GM (2020) Natural products as sources of new drugs over the nearly four decades from 01/1981 to 09/2019. J Nat Prod 83(3): 770-803. https://doi.org/10.1021/acs.jnatprod.9b01285

2. Nguyen $\mathrm{NH}$, Ta QTH, Pham QT, Luong TNH, Phung VT, Duong TH, Vo VG (2020) Anticancer activity of novel plant extracts and compounds from Adenosma bracteosum (Bonati) in human lung and liver cancer cells. Molecules 25(12):2912. https://doi.org/10.3390/molecules25122912

3. Veeresham C (2012) Natural products derived from plants as a source of drugs. J Adv Pharm Technol Res 3(4):200-201. https://doi.org/10.4103/22314040.104709

4. Kinghorn AD, Pan L, Fletcher JN, Chai H (2011) The relevance of higher plants in lead compound discovery programs. J Nat Prod 74(6):1539-1555. https://doi.org/10.1021/np200391c

5. Cragg GM, Newman DJ $(2013,1830)$ Natural products: a continuing source of novel drug leads. Biochim Biophys Acta (6):3670-3695. https://doi.org/10. 1016/j.bbagen.2013.02.008

6. Ifeoma O, Oluwakanyinsola S (2013) Screening of herbal medicines for potential toxicities. In: Gowder SJT (ed) New insights into toxicity and drug testing. London: IntechOpen Limited; pp 83-88. https://doi.org/10.5772/ 54493

7. Vinaykumar NM, Mahmood R, Krishna V, Ravishankara B, Shastri SL (2020) Antioxidant and in vivo hepatoprotective effects of Gardenia gummifera L.f. fruit methanol extract. Clin Phytosci 6(47). https://doi.org/10.1186/s40816020-00188-7

8. Bode AM, Dong Z (2015) Toxic phytochemicals and their potential risks for human cancer. Cancer Prev Res (Phila) 8(1):1-8 https://doi.org/10.1158/ 19406207.CAPR-14-0160

9. Aamir K, Khan HU, Hossain CF, Afrin MR, Shaik I, Salleh N, Giribabu N, Arya A (2019) Oral toxicity of arjunolic acid on hematological, biochemical and histopathological investigations in female Sprague Dawley rats. Peer J 22(7): e8045. https://doi.org/10.7717/peerj.8045

10. Salehi B, Fokou PVT, Yamthe LRT, Tali BT, Adetunji CO, Rahavian A, Mudau FN, Martorell M, Setzer WN, Rodrigues CF, Martins N, Cho WC, Sharifi-Rad J (2019) Phytochemicals in prostate cancer: from bioactive molecules to upcoming therapeutic agents. Nutrients 11(7):1483. https://doi.org/10.3390/ nu11071483

11. Vysakh A, Jayesh K, Helen LR, Jyothis M, Latha MS (2020) Acute oral toxicity and anti-inflammatory evaluation of methanolic extract of Rotula aquatica roots in Wistar rats. J Ayurveda Integr Med 11(1):45-52. https://doi.org/10. 1016/j.jaim.2017.09.007

12. Sevimli FK, Kozan E, Sevimli A, Dogan N, Bulbul A (2009) The acute effects of single-dose orally administered doramectin, eprinomectin and selamectin on natural infections of Syphacia muris in rats. Exp Parasitol 122(3):177-181. https://doi.org/10.1016/j.exppara.2009.03.008

13. Sylla B, Lavoie S, Legault J, Gauthier C, Pichette A (2019) Synthesis, cytotoxicity and anti-inflammatory activity of rhamnose-containing ursolic and betulinic acid saponins. RSC Adv 9(68):39743-39757. https://doi.org/10 1039/C9RA09389C

14. Tsai JC, Peng WH, Chiu TH, Lai SC, Lee CY (2011) Anti-inflammatory effects of Scoparia dulcis L. and betulinic acid. Am J Chin Med 39(5):943-956. https://doi.org/10.1142/S0192415X11009329
15. Pavlova NI, Savinova OV, Nikolaeva SN, Boreko El, Flekhter OB (2003) Antiviral activity of betulin, betulinic and betulonic acids against some enveloped and non-enveloped viruses. Fitoterapia 74(5):489-492. https:// doi.org/10.1016/s0367-326x(03)00123-0

16. Steele JC, Warhurst DC, Kirby GC, Simmonds MS (1999) In vitro and in vivo evaluation of betulinic acid as an antimalarial. Phytother Res 13(2):115-119. https://doi.org/10.1002/(SICI)1099-1573(199903)13:2\%3C115:.AID-PTR404\% 3E3.0.CO;2-1

17. Alakurtti S, Makela T, Koskimies S, Yli-Kauhaluoma J (2006) Pharmacological properties of the ubiquitous natural product betulin. Eur J Pharm Sci 29(1): 1-13. https://doi.org/10.1016/j.ejps.2006.04.006

18. Enwerem NM, Okogun II, Wambebe CO, Okorie DA, Akah PA (2001) Anthelmintic activity of the stem bark extracts of Berlina grandiflora and one of its active principles, Betulinic acid. Phytomed 8(2):112-114. https://doi. org/10.1078/0944-7113-00023

19. Jäger S, Laszczyk MN, Scheffler A (2008) A preliminary pharmacokinetic study of Betulin, the main pentacyclic triterpene from extract of outer bark of Birch (Betulae alba cortex). Molecules 13(12):3224-3235. https://doi.org/ 10.3390/molecules13123224

20. Mullauer FB, van Bloois L, Daalhuisen JB, Brink MST, Storm G, Medema JP, Schiffelers RM, Kessler JH (2011) Betulinic acid delivered in liposomes reduces growth of human lung and colon cancers in mice without causing systemic toxicity. Anti-Cancer Drugs 22(3):223-233. https://doi.org/10.1097/ CAD 0b013e3283421035

21. Saraswat B, Visen PKS, Agarwal DP (2000) Ursolic Acid isolated from Eucalyptus tereticornis protects against ethanol toxicity inisolated rat hepatocytes. Phytother Res 14(3):163-166. https://doi.org/10.1002/(sici)10991573(200005)14:3<163:aid-ptr588>3.0.co:2-d

22. Liu J (1995) Pharmacology of oleanolic acid and ursolic acid. J Ethnopharmacol 49(2):57-68. https://doi.org/10.1016/0378-8741(95)90032-2

23. Huang MT, Ho CT, Wang ZY, Ferraro T, Lou YR, Stauber K, Ma W, Georgiadis C, Laskin JD, Conney AH (1994) Inhibition of skin tumorigenesis by rosemary and its constituents camosol and ursolic acid. Cancer Res 54(3):701-708

24. Cha HJ, Bae SK, Lee HY, Lee OH, Sato H, Seiki M, Park BC, Kim KW (1996) Anti-invasive activity of ursolic acid correlates with the reduced expression of matrix metalloproteinase-9 (MMP-9) in HT1080 human fibrosarcoma cells. Cancer Res 56(10):2281-2284

25. Saini P, Gayen P, Kumar D, Nayak A, Mukherjee N, Mukherjee S, Pal BC, Babu SPS (2014) Antifilarial effect of ursolic acid from Nyctanthes arbortristis: molecular and biochemical evidences. Parasitol Int 63(5):717-728. https:// doi.org/10.1016/j.parint.2014.06.008

26. Wilkinson K, Boyd JD, Glicksman M, Moore KJ, El Khoury J (2011) A high content drug screen identifies ursolic acid as an inhibitor of amyloid beta protein interactions with its receptor CD36. J Biol Chem 286(40):3491434922. https://doi.org/10.1074/jbc.M111.232116

27. Geerlofs L, He Z, Xiao S (2020) Xiao ZC (2020) Repeated dose (90 days) oral toxicity study of ursolic acid in Han-Wistar rats. Toxicol Rep 7:610-623. https://doi.org/10.1016/j.toxrep.2020.04.005

28. Wang XH, Zhou SY, Qian ZZ, Zhang HL, Qiu LH, Song Z, Zhao J, Wang P, Hao XS, Wang HQ (2013) Evaluation of toxicity and single-dose pharmacokinetics of intravenous ursolic acid liposomes in healthy adult volunteers and patients with advanced solid tumors. Expert Opin Drug Metab Toxicol 9(2):117-125. https://doi.org/10.1517/17425255.2013. 738667

29. Shai LJ, Bizimenyera ES, Bagla V, McGaw LJ, Eloff JN (2009) Curtisia dentata (Cornaceae) leaf extracts and isolated compounds inhibit motility of parasitic and free-living nematodes. Ondesterpoort J Vet Res 76(2):249-256. https://doi.org/10.4102/ojvr.v76i2.49

30. Vijaya YAK, Gogoi S (2018) In vitro and in vivo anthelmintic efficacy of two pentacyclic triterpenoids, ursolic acid and betulinic acid against mice pinworm, Syphacia obvelata. J Parasit Dis 42(1):144-149. https://doi.org/10. 1007/s12639-017-0960-0

31. Vijaya YAK (2016) In vitro anthelmintic assessment of selected phytochemicals against Hymenolepis diminuta, a zoonotic tapeworm. J Parasit Dis 40(3):1082-1086. https://doi.org/10.1007/s12639-014-0560-1

32. Yadav AK, Temjenmongla (2011) Anticestodal activity of Houttuynia cordata leaf extract against Hymenolepis diminuta in experimentally infected rats. J Parasit Dis 35(2):190-194. https://doi.org/10.1007/s12639011-0050-7

33. OECD 423 (2001) Acute Oral Toxicity-Acute Toxic Class Method, OECD Guidelines for the Testing of Chemicals, Section 4, Organization for 
Economic Cooperation and Development. OECD Publishing, Paris. https:// doi.org/10.1787/9789264071001-en

34. OECD 407 (2008) Repeated Dose 28-day Oral Toxicity Study in Rodents, OECD Guidelines for the Testing of Chemicals, Section 4, Organization for Economic Cooperation and Development. OECD Publishing, Paris. https:// doi.org/10.1787/9789264070684-en

35. Selvakumar GP, Manivasagam T, Rekha KR, Jayaraj RL, Elangovan N (2015) Escin, a novel triterpene, mitigates chronic MPTP/p-induced dopaminergic toxicity by attenuating mitochondrial dysfunction, oxidative stress, and apoptosis. J Mol Neurosci 55(1):184-197 https://doi.org/10.1007/s12031-0140303-x

36. Lu J, Guan S, Liu JB (2009) Acute and genetic toxicity of ursolic acid extract from Ledum pulastre L. Food Sci 30(13):250-252. https://doi.org/10.7506/ spkx10026630200913057

37. Gatsing D, Kamsu GT, Fodouop SPC, Tagne RS, Kodjio N, Fakam ALN (2019) Evaluation of the acute and sub-chronic toxicity of the ethanolic extract of Curcuma longa (Zingiberaceae) in Wistar albino rats. Mod Chem Appl 7(1): 267. https://doi.org/10.35248/2329-6798.19.7.267

38. Robinson S, Chapman K, Hudson S, Sparrow S, Spencer-Briggs D, Danks A, Hill R, Everett D, Mulier B, Old S, Bruce C (2009) Guidance on dose level selection for regulatory general toxicology studies for pharmaceuticals. National Centre for the Replacement, Refinement and Reduction of Animals in Research. Laboratory Animal Science Association

39. Akpabio CJ, Efuruibe AA, Adeleke GE, Ogunsola MO, Maduagwu EN (2013) $\mathrm{N}$-Nitrosation of dimethylamine hydrochloride and its toxicology in the Wistar rats fed different levels of dietary protein. Eur J Exp Biol 3(2):94-103

40. Konan NA, Bachhi EM, Lincopan N, Varela SD, Varnada EA (2007) Acute, subacute toxicity and genotoxic effect of a hydroethanolic extract of the cashew (Anacardium occidentale L.). J Ethnopharmacol 110(1):30-38. https:// doi.org/10.1016/j.jep.2006.08.033

41. Mukinda JT, Syce JA (2007) Acute and chronic toxicity of the aqueous extract of Artemisia afra in rodents. J Ethnopharmacol 112(1):138-144. https://doi.org/10.1016/j.jep.2007.02.011

42. Hosseinzadeh H, Shakib SS, Sameni AK, Taghiabadi E (2013) Acute and subacute toxicity of safranal, a constituent of saffron, in mice and rats. Iran J Pharm Res 12:93-99. https://doi.org/10.1016/j.clinbiochem.2011.08.1100

43. Nigatu TA, Afework M, Urga K, Ergete W, Makonnen E (2017) Toxicological investigation of acute and chronic treatment with Gnidia stenophylla Gilg root extract on some blood parameters and histopathology of spleen, liver and kidney in mice. BMC Res Notes 10:625. https://doi.org/10.1186/s13104017-2964-3

44. Wang W, Dong Z, Zhang J, Zhou X, Wei X, Cheng F, Li B, Zhang J (2019) Acute and subacute toxicity assessment of oxyclozanide in Wistar rats. Front Vet Sci 6(249). https://doi.org/10.3389/fvets.2019.00294

\section{Publisher's Note}

Springer Nature remains neutral with regard to jurisdictional claims in published maps and institutional affiliations.

\section{Submit your manuscript to a SpringerOpen ${ }^{\circ}$ journal and benefit from:}

- Convenient online submission

- Rigorous peer review

- Open access: articles freely available online

- High visibility within the field

- Retaining the copyright to your article

Submit your next manuscript at $\boldsymbol{\nabla}$ springeropen.com 